\title{
Complement of Source Equation of Elastic Line
}

\author{
Mirjana Filipovic • Miomir Vukobratovic
}

Received: 15 May 2007 / Accepted: 28 January 2008 /

Published online: 16 April 2008

(C) The Author(s) 2008

\begin{abstract}
A new notion of joint, defined in terms of the state of motor (active or locked) and type of the elastic or rigid element, gear and/or link that follows after the motor, is introduced. Special attention is paid to the motion of the flexible links in the robotic configuration. The paper deals with the relationship between the equation of elastic line equilibrium, the "Euler-Bernoulli approach" (EBA), and equation of motion at the point of elastic line tip, the "Lumped-mass approach" (LMA). The Euler-Bernoulli equations (which have for a long time been used in the literature) should be expanded according to the requirements of the motion complexity of elastic robotic systems. The Euler-Bernoulli equation (based on the known laws of dynamics) should be supplemented with all the forces that are participating in the formation of the elasticity moment of the considered mode. This yields the difference in the structure of Euler-Bernoulli equations for each mode. The stiffness matrix is a full matrix. Mathematical model of the actuators also comprises coupling between elasticity forces. Particular integral of Daniel Bernoulli should be supplemented with the stationary character of elastic deformation of any point of the considered mode, caused by the present forces. General form of the elastic line is a direct outcome of the system motion dynamics, and cannot be described by one scalar equation but by three equations for position and three equations for orientation of every point on that elastic line. The choice of reference trajectory is analyzed. Simulation results are shown for a selected robotic example involving the simultaneous presence of elasticity of the gear and of the link (two modes), as well as the environment force dynamics.
\end{abstract}

Keywords Robot $\cdot$ Modeling $\cdot$ Elastic deformation $\cdot$ Gear $\cdot$ Link $\cdot$ Coupling $\cdot$ Dynamics $\cdot$ Kinematics $\cdot$ Trajectory planning

\section{Introduction}

Modeling and control of elastic robotic systems has been a challenge to researchers in the last three decades. In [34], the control of robots with elastic joints in contact with dynamic 
environment is considered. In [4], the feedback control was formed for the robot with flexible links (two-beam, two-joint systems) with distributed flexibility, robots with flexible links being also dealt with in [14]. In paper [27] a nonlinear control strategy for tip position trajectory tracking of a class of structurally flexible multi-link manipulators is developed. Authors of papers [25] and [24] derived dynamic equations of the joint angle, the vibration of the flexible arm, and the contact force.

The paper [35] presents an approach to end point control of elastic manipulators based on the nonlinear predictive control theory. $[31,32]$ present a method for the generation of efficient kinematics and dynamic models of flexible robots. In [30] author discusses the force control problem for flexible joint manipulators.

In paper [13] the authors extend the integral manifold approach for the control of flexible joint robot manipulators from the known parameter case to the adaptive case. The author of paper [18] designed a control law for local regulation of contact force and position vectors to desired constant vectors. In paper [6] differently from conventional approaches, authors focus on the design of rigid part motion control and the selection of bandwidth of rigid subsystem. Paper [20] presents the derivation of the equations of motion for application to mechanical manipulators with flexible links. In [21] the equations are derived using Hamilton's principle, and are nonlinear integro-differential equations. Method of separation of variables and the Galerkin's approach are suggested in paper [22] for the boundary-value problem with time-dependent boundary condition.

Mathematical model of a mechanism with one degree of freedom (DOF), with one elastic gear was defined by Spong [29] as early as in 1987.Based on the same principle, elasticity of gears is introduced into the mathematical model in this paper, as in paper [1012] also. However, when the introduction of link flexibility into the mathematical model is concerned, it is necessary to point to some essential problems in this domain.

LMA is a method which defines equilibrium equation (motion equation) of any point of considered mechanism. If any link of the mechanism is elastic then we also can define motion equation of any point of presented link. We don't know exactly when this approach has been stated. It defines dynamic equation in any point of mechanism during movement. The LMA (used in [2-5]) gives the possibility to analyze the motion of the any point of each mode. Papers with this research topic (approach) were rare in robotics journals in the last two decades.

EBA assumes the use of Euler-Bernoulli equations which appeared in 1750. EBA (used in $[7-9,15-17,23,25,31,32]$ etc.) gives the possibility to analyze a flexible line form of each mode in the course of task realization. (See Fig. $1 ; \varepsilon_{i j}$ is the flexibility moment defining flexural deformation of the considered body.)

The EBA is an approach that is still in the focus of researchers' interest and it was analyzed most often in the last decades.

In the pertinent literature no relationship has been established between the LMA and EBA.

We consider that EBA and LMA, are two comparative methods addressing the same problem but from different aspects $[11,12]$.

We consider them as two comparable methods considering the same problem but from different aspects. Using the EBA we obtain the equations of flexible line model of each mode and by setting boundary conditions we obtain model equations of motion at the point of the tip (or any other point) of each mode, which is in fact the LMA. These equations are of different types and can't be combined. As the equation of motion for the mode tip point is essentially LMA and it follows directly from the equation of flexible line obtained via EBA for the preset boundary conditions, it clearly comes out 
Fig. 1 LMA and EBA as two comparative approaches

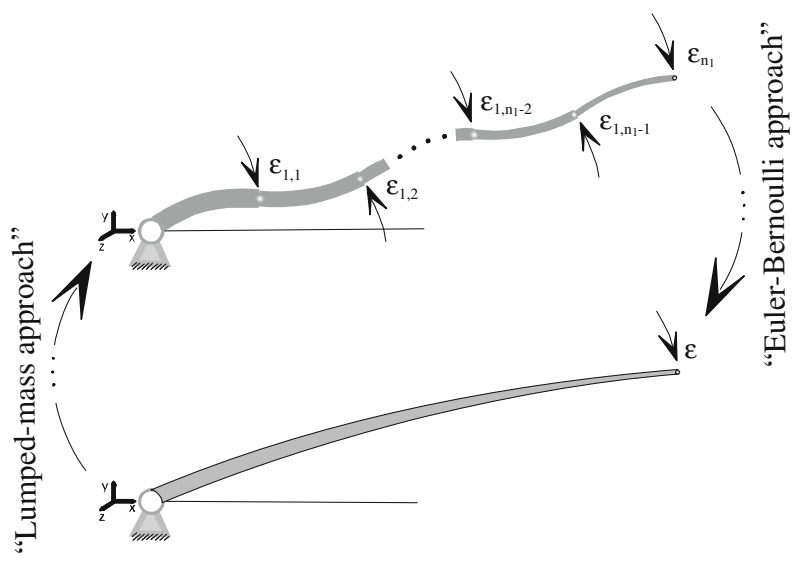

that the structures of these equations are the same (whereas the content of elements of these structures is not the same). Just for this reason, each of the two methods can be used as a check of validity of the other (assuming that no crude approximations have been made).

Mathematical model obtained by any of the methods should satisfy elementary structure of the models of elastic mechanisms known in the literature [33]. This has been treated in detail in our work, but it is not the only essential problem existing in the pertinent literature.

In the previous literature $[7-9,15,16,26]$ the general solution of the motion of an elastic robotic system has been obtained by considering flexural deformations as transversal oscillations that can be determined by the method of particular integrals of D. Bernoulli.

It is known that flexural deformations of a body can be caused by:

- disturbance forces, causing an oscillatory motion,

- stationary forces, causing the motion of a stationary character.

We consider that any elastic deformation can be presented by superimposing D. Bernoulli's particular solutions of the oscillatory character and stationary solution of the forced character. See papers $[11,12]$.

The first detailed presentation of the procedure for creating a reference trajectory was given in [1] and later in [8].

In our work we have synthesized a reference trajectory for a robot model including elastic gears and links and the presence of environment force. The reference trajectory is calculated from the overall dynamic model, when the robot tip is tracking a desired trajectory in a reference regime in the absence of disturbances.

Elastic deformation (of flexible links and elastic gears) is a quantity which is at least partly encompassed by the reference trajectory. It is assumed that all elasticity characteristics in the system (both of stiffness and damping) are "known", at least partly and at that level can be included into the process of defining the reference motion. The reference trajectory thus defined allows the possibility of applying very simple control laws via PD local feedback loops, which ensures reliable tracking of the robotic tip considered in the space of Cartesian coordinates to the level of known elasticity parameters, too.

As far as the working regime of the robot is concerned we think that all forces should participate in generating elastic deformations and that it is a crude approximation to assume 
that elastic effects are generated only by gravitational force, or only by the environment force as in $[23,25]$, or that Coriolis and centrifugal forces can be neglected altogether that elastic deviations are so small, so that inertia matrix is not dependent on them, as assumed in [17].

"Assumed modes technique" appeared in 1967 and was proposed by Meirovitch in [26]. This technique was used by all authors in the last 40 years to form Euler Bernoulli equation. In our paper we form Euler Bernoulli equation but we do not use "assumed modes technique" in contrast to our contemporaries. We assume that the elastic deformation, and also circular frequency of each mode of elastic element, is a consequence of the overall dynamics motion of the robotic system.

Let us emphasize once again that in this paper we propose a mathematical model solution that includes in its root the possibility for analyzing simultaneously both present phenomena - the elasticity of gears and the flexibility of links, and the idea originated from [5], but not on the same principles. We show how the continuously present environment dynamics force affects the behaviour of an elastic robot system.

Section 2 defines the kinematics model (types of joints, direct and inverse kinematics). Section 3 defines the dynamics model of elastic robotic systems. Subsection 3.1 gives the interpretation of the source equations of elastic line. A supplement to the source equations of flexible line is given. In Subsections 3.2, 3.3 and 3.4 we define a general form of the equation of flexible line of a complex robotic system of arbitrary configuration, using EBA. The flexible line equation is extended by a damping component. Also, we demonstrate that the particular integral of D. Bernoulli is just a component of flexural deformation of any point of the mode considered, to which it is necessary to add the component of flexural deformation of the stationary regime. Section 4 demonstrates the relationship between the equation of elastic line motion and equation of motion at any point of the elastic line. Section 5 analyzes the choice of reference trajectory. Section 6 analyzes the movement dynamics of a multiple DOF elastic robotic pair with elastic gear and flexible link in the presence of the second mode and environment force. Section 7 gives some concluding remarks.

\section{Kinematics}

Kinematics and dynamics of a robotic system are analyzed. Since elasticity elements are introduced, it is necessary to explain in detail, first of all, the kinematics of these systems in order to have dynamic modeling as efficient as possible.

In the presence of elasticity elements, the notion of a joint (degree-of-freedom, DOF) requires a new meaning, which is necessary to expand and explain. We will define the types of rigid and elastic elements that may appear in a robotic configuration. The joint type is defined as a combination of rigid or elastic gear and/or rigid or flexible link and/or the motor that is directly in front of these elements. In its configuration, each joint may contain:

- motor and/or

- gearing system and/or

- link.

The motor may be:

- Active, if it realizes the motion whereby the motor deflection angle $\bar{\theta}_{i} \neq$ const or 
- Locked, if it is fixed at a certain position, and its speed and acceleration are zero, then $\bar{\theta}_{i}=$ const .

Behind any motor there may be implemented a gearing system, which may be:

- Elastic, if the deformation is elastic, rigidity characteristic is $C_{\xi i}[\mathrm{Nm} / \mathrm{rad}]$ and damping characteristic is $B_{\xi i}[\mathrm{Nm} /(\mathrm{rad} / \mathrm{s})]$, then the gear deflection angle $\xi_{i} \neq 0$.

- Rigid, if elastic deformation is $\xi_{i}=0$.

Behind any motor and/or gear there may be implemented a link, which may be:

- Elastic (Flexible), rigidity characteristic is $C_{S i}[\mathrm{~N} / \mathrm{m}]$ and damping characteristic is $B_{s i}[N . s / m]$, and then the link bending angle $\vartheta_{i} \neq 0$.

- Rigid, if flexural deformation is $\vartheta_{i}=0$.

This means that a joint can be defined in dependence of the working state of the motor, type of gearing and type of link. Let $\aleph_{i}$ be the elastic deformation of any type of the elastic element after the motor. We define four types of joints, namely $\mathrm{AE}_{\aleph}, \mathrm{LE}_{\aleph}, \mathrm{AR}$ and CR.

Type $A E_{\aleph}$ is characterized by an active motor and an arbitrary elastic element (or more of them in a series) behind the motor, where elastic deformation takes place in the direction of motor motion. The overall coordinate $q_{i}$ contains the following components: $\bar{\theta}_{i}-$ motor rotation angle and $\aleph_{e i}$-elastic deformation of the elastic element behind of the motor (or $\sum \aleph_{e i}$, the sum of elastic deformations of the elastic elements in the series coming after the motor).

All these angles vary in the course of robotic task realization.

$$
q_{i}=\bar{\theta}_{i}+\sum \aleph_{e i}
$$

This will be explained in more detail on the examples. If the motor is active, the gear elastic (whereby elastic deformation takes place in the direction of motor motion) and the

Fig. 2 Spatial sketch of a joint of the type $\mathrm{AE} \xi$ and its geometry for an elastic gear and rigid link
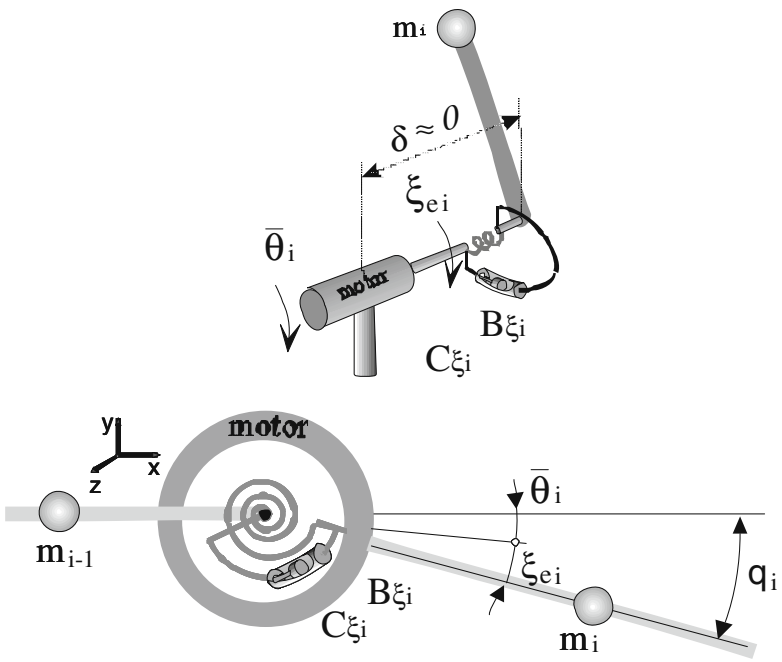
link rigid, then this type of joint will be denoted shortly as $\mathrm{AE} \xi$. In Fig. 2 is shown a spatial sketch of the joint of the AE $\xi$ type, as well as its geometry.

The angle $q_{i}$ contains the following components: $\bar{\theta}_{i}$ and $\xi_{e i}-$ the joint deflection angle.

$$
q_{i}=\bar{\theta}_{i}+\xi_{e i} .
$$

If the gear is rigid and the link behind it flexible, as in Fig. 3, which has elastic deformation taking place only in the direction of the motor shaft deflection, then such a joint is defined as being of type $\mathrm{AE}_{\vartheta}$. The overall coordinate $q_{i}$ contains the following components: $\bar{\theta}_{i}$ and $\vartheta_{e i}$ - the link bending angle. The angle $\vartheta_{e i}$ can also vary in the course of robotic task realization.

$$
q_{i}=\bar{\theta}_{i}+\vartheta_{e i} .
$$

A joint is also of the AE $\xi$ type if the gear and link behind the motor are elastic and if both elastic deformations take place in the direction of motor shaft deflection. It bears the designation of that elastic element that is directly behind the motor (see Fig. 4).

The overall coordinate $q_{i}$ contains the following components:

$$
q_{i}=\bar{\theta}_{i}+\xi_{e i}+\vartheta_{e i}
$$

It is clear that these are only special cases, when elastic deformation takes place in the direction of motor shaft deflection. Because of that it is important to define also a "kinematics" connection for the case when elastic deformation of the gear or link is not

Fig. 3 Spatial sketch of a joint of the type $\mathrm{AE} \vartheta$ and its geometry for a rigid gear and flexible link

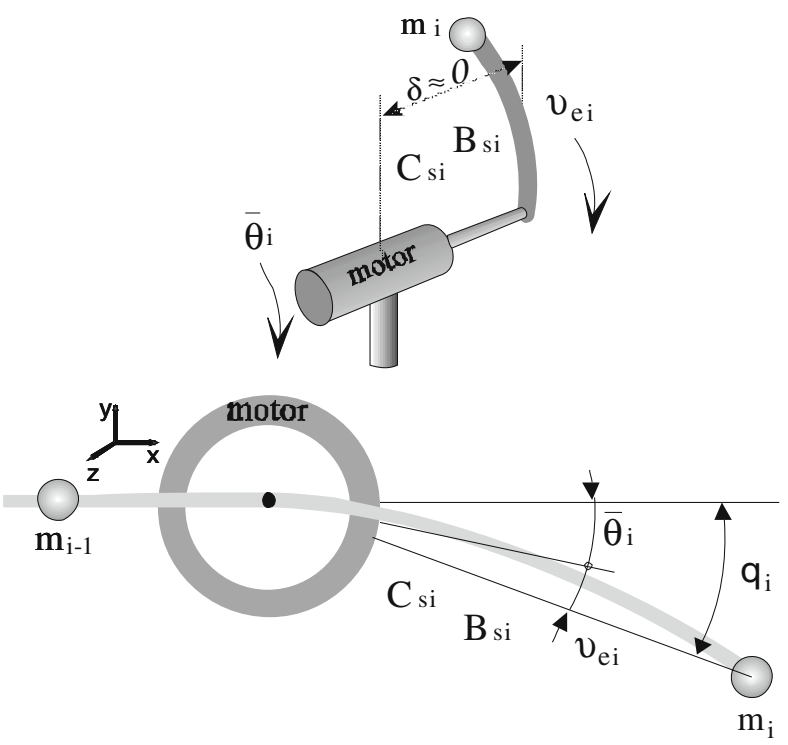



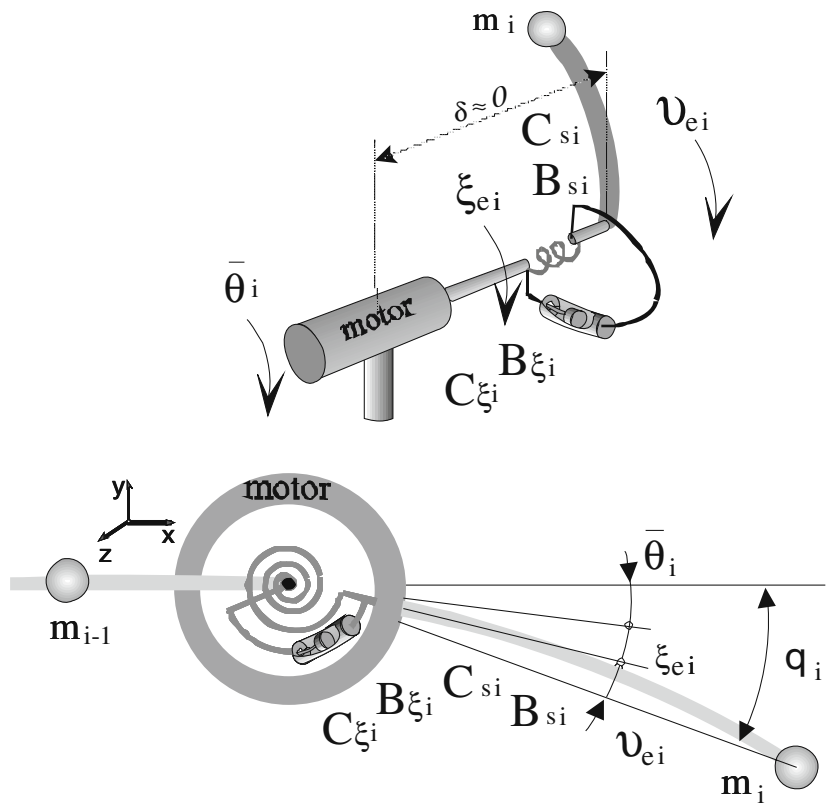

Fig. 4 Spatial sketch of a joint of the type $\mathrm{AE} \xi$ and its geometry for an elastic gear and flexible link

taking place in the direction of motor shaft deflection or, if there is no a drive in front of the elastic deformation (that is in front of the elastic element).

Type $L E_{\aleph}$ is characterized by a locked motor and an arbitrary elastic element (or more of them in a series) behind the locked motor.

In fact, a locked motor has no an actuator's function. It is present only as a mass. For this reason the same designation $\mathrm{LE}_{\aleph}$ is used when the motor either exists or is missing, but there is only one elastic element (or more of them in a series, whose deformation takes place in the same direction).

The overall coordinate $q_{i}$ contains elastic deformation $\aleph_{n i}$ of the elastic element (or sum of elastic deformations $\sum_{k=1}^{c} \aleph_{n i, k}$ of the elastic elements in a series that take place in the same direction). All these quantities of elastic deformations vary in the course of robotic task realization.

$$
q_{i}=\sum_{k=1}^{c} \aleph_{n i, k} .
$$

Let us explain this in more detail on the examples. If the motor is locked, the gear elastic and the link rigid, then this type of joint is denoted as LE $\xi$. In Fig. 5 is shown a geometric depiction of a joint of the LE $\xi$ type, as well as of the same type of joint with elastic gear and rigid link. The overall coordinate $q_{i}$ contains only $\xi_{n i}$.

$$
q_{i}=\xi_{n i}
$$


Fig. 5 Spatial sketch of a joint of the type $\mathrm{LE} \xi$ and its geometry for an elastic gear and rigid link

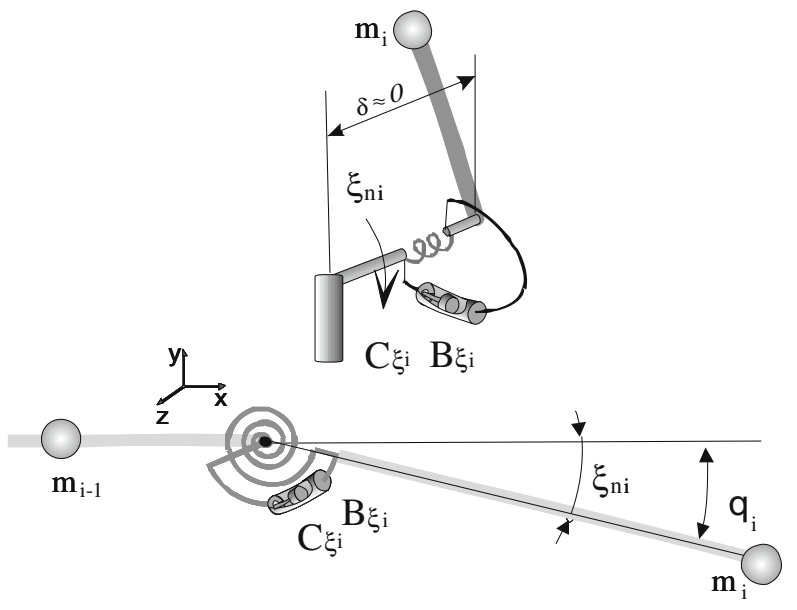

A joint of the LE $\vartheta$ type, involving a rigid gear and a flexible link, is shown in Fig. 6. The overall coordinate $q_{i}$ contains only $\vartheta_{n i}$.

$$
q_{i}=\vartheta_{n i}
$$

It is also possible to define another type of joint $\mathrm{LE} \xi$, whereby the gear is elastic and the link is flexible, shown in Fig. 7. The joint bears the designation of that elastic element which comes first in the sequence. The overall coordinate $q_{i}$ contains only the sum of elastic deformations $\xi_{n i}$ and $\vartheta_{n i}$.

$$
q_{i}=\xi_{n i}+\vartheta_{n i}
$$

Type $A R$ is characterized by an active motor and all rigid elements that come after the motor (both the gear and link).

Fig. 6 Spatial sketch of a joint of the type LE $\vartheta$ and its geometry for a rigid gear and flexible link

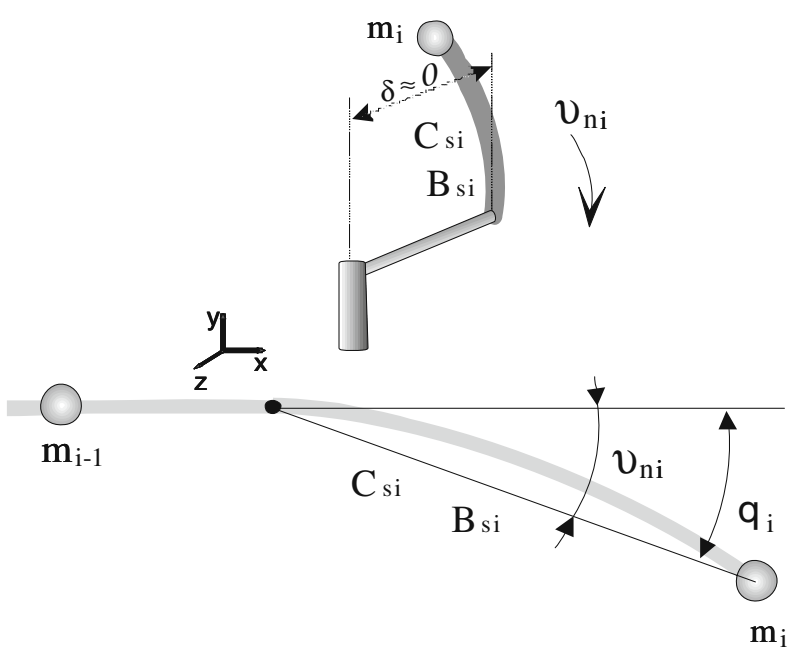


Fig. 7 Spatial sketch of a joint of the type $\mathrm{LE} \xi$ and its geometry for an elastic gear and flexible link

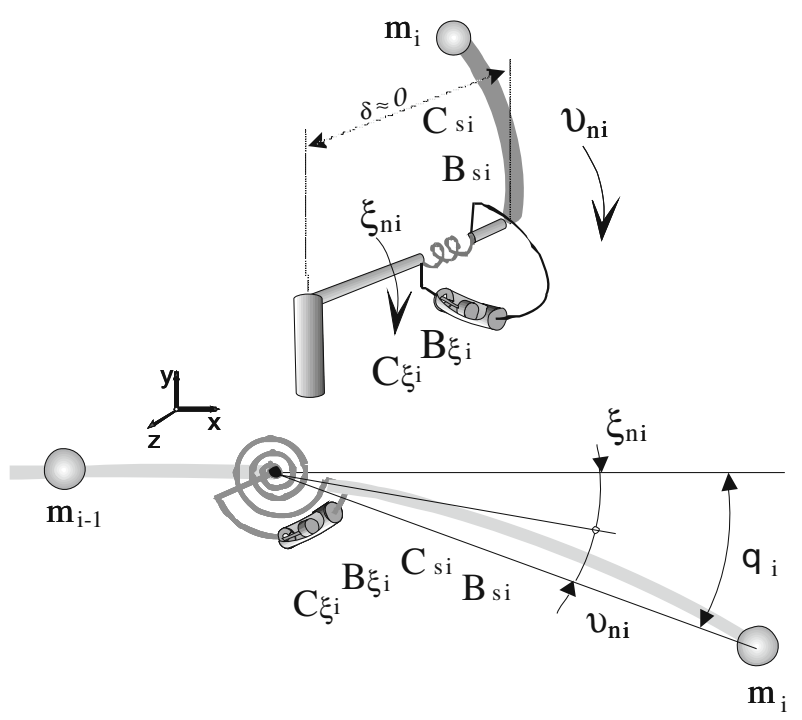

In this case, the overall coordinate $q_{i}$ is equal to the motor rotation angle $\bar{\theta}_{i}$.

$$
q_{i}=\bar{\theta}_{i}
$$

Type $L R$ is characterized by a locked motor, a rigid gear after it, and a rigid link.

$$
q_{i}=0
$$

By defining the type of every joint in the branched chain of the robotic system we define the configuration of that system, which is a prerequisite for defining its mathematical model and analyzing its dynamic behavior.

Therefore, in this paper we analyze the behavior of a robotic system which may contain joints of the type $A E_{\aleph}, L E_{\aleph}, A R$ and $L R$.

The rotation matrix which describes the change of the position (Cartesian coordinates) and orientation (Euler angles) of the tip of every link (or mode of segment) is of the form:

$$
T_{e_{i}}^{i-1}=\left[\begin{array}{cccc}
\cos q_{i} & -\sin q_{i} \cdot \cos \alpha_{i} & \sin q_{i} \cdot \sin \alpha_{i} & l_{i} \cdot \cos q_{i} \\
\sin q_{i} & \cos q_{i} \cdot \cos \alpha_{i} & -\cos q_{i} \cdot \sin \alpha_{i} & l_{i} \cdot \sin q_{i} \\
0 & \sin \alpha_{i} & \cos \alpha_{i} & d_{i} \\
0 & 0 & 0 & 1
\end{array}\right]
$$

Where $\alpha_{i}, l_{i}$ and $d_{i}$ are the Denavit-Hartenberg parameters. The overall transformation matrix is of the form:

$$
T_{e_{n}}^{0}=T_{e_{1}}^{0} \cdot T_{e_{2}}^{1} \cdot T_{e_{3}}^{2} \cdots T_{e_{i}}^{i-1} \cdots T_{e_{n}}^{n-1}
$$


The Jacobi matrix for a manipulator with elastic joints maps the velocity vector of the external coordinates $\dot{p}_{s}$ into the velocity vector of internal coordinates $\dot{q}$ :

$$
\dot{q}=J_{e}^{-1}(q) \cdot \dot{p}_{s}
$$

Where $\dot{p}_{s}=\left[\begin{array}{llllll}\dot{x} & \dot{y} & \dot{z} & \dot{\psi} & \dot{\wp} & \dot{\varphi}\end{array}\right]^{T}$ defines the velocity of a given point of the robotic system in the Cartesian coordinates, whereas $\dot{q}=\left[\begin{array}{llllll}\dot{q}_{1} & \dot{q}_{2} & \dot{q}_{3} & \dot{q}_{4} & \ldots & \dot{q}_{n}\end{array}\right]^{T}$ defines the velocity vector of internal coordinates of the joints defined by Eqs. 1-10. Elements of the Jacobian are only functions of the elements of the homogenous transformation matrix $T_{e n}^{0}$. It is clear that each branched chain in the complex mechanism has its finite transformation matrix, as well as its Jacobi matrix.

\section{Dynamics}

3.1 Interpretation of the Source Equations of Elastic Line

Equation of the elastic line of beam bending is of the following form:

$$
\hat{M}_{1,1}+\beta_{1,1} \cdot \frac{\partial^{2} \hat{y}_{1,1}}{\partial \hat{x}_{1,1}^{2}}=0
$$

i.e.

$$
\begin{gathered}
\hat{M}_{1,1}+\hat{\varepsilon}_{1,1}=0 . \\
\hat{\varepsilon}_{1,1}=\beta_{1,1} \cdot \frac{\partial^{2} \hat{y}_{1,1}}{\partial \hat{x}_{1,1}^{2}} .
\end{gathered}
$$

where $\widehat{M}_{1,1}[\mathrm{Nm}]$ is the load moment, in these source equations encompassing only inertia, $\widehat{\varepsilon}_{1,1}$ bending moment, $\beta_{1,1}\left[\mathrm{Nm}^{2}\right]$ is the flexural rigidity.

General solution of motion, i.e. the form of transversal oscillations of flexible beams can be found by the method of particular integrals of $\mathrm{D}$. Bernoulli, that is:

$$
\begin{gathered}
\hat{y}_{t o 1,1}\left(\widehat{x}_{1,1}, t\right)=\widehat{X}_{1,1}\left(\widehat{x}_{1,1}\right) \cdot \widehat{T}_{t o 1,1}(t) . \\
\widehat{X}_{1,1}\left(\widehat{x}_{1,1}\right)=C_{1,(1,1)} \cos k_{1,1} \widehat{x}_{1,1}+C_{2,(1,1)} \sin k_{1,1} \widehat{x}_{1,1}+. \\
+C_{3,(1,1)} C h k_{1,1} \widehat{x}_{1,1}+C_{4,(1,1)} S h k_{1,1} \widehat{x}_{1,1} \\
\widehat{T}_{t o 1,1}(t)=A_{1,1} \cos p_{1,1} t+B_{1,1} \sin p_{1,1} t .
\end{gathered}
$$


Fig. 8 Idealized motion of elastic body according to

D. Bernoulli.

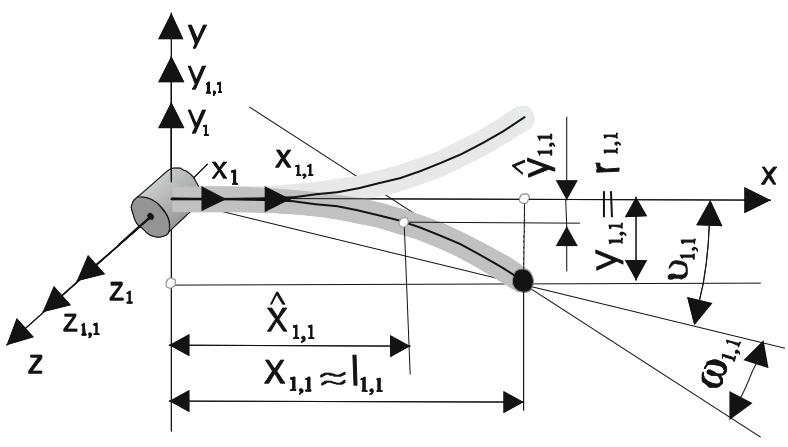

See Fig. 8. By superimposing the particular solutions (17), any transversal oscillation can be presented in the following form:

$$
\widehat{y}_{t o 1}\left(\widehat{x}_{1, j}, t\right)=\sum_{j=1}^{\infty} \widehat{X}_{1, j}\left(\widehat{x}_{1, j}\right) \cdot \widehat{T}_{t o 1, j}(t) .
$$

$x_{1,1}, y_{1,1}, z_{1,1}$ - is a local coordinate frame, which is set in the base of considered mode. In this case (see Fig. 8.) this is the first mode of the first link, while in a general case this is $x_{i, j}$, $y_{i, j}, z_{i, j}$, where $j$ is the serial number of the mode of considered link $j=1,2,3 \ldots n_{i}$, and $i$ is the serial number of the link of the considered robotic system $i=1,2,3 \ldots m$.

$x_{1}, y_{1}, z_{1}$ is a local coordinate frame, which is set in the base of the considered link. In a general case it is $x_{i}, y_{i}, z_{i}$.

$x, y, z-$ is the basic coordinate frame, which is set in the root of the considered robotic system.

$\vartheta_{1,1}$ is the bending angle of the first mode of the first link. $\omega_{1,1}$ is the rotation angle of the top of the same mode (see [19]).

Remark I: Equations 14-20 need a short explanation that, we think, should be assumed, but which is missing from the original literature [33]. Euler and Bernoulli wrote Eq. 20 based on 'vision'. They did not define the mathematical model of a link with an infinite number of modes, which has a general form of Eq. 21, but they did define the motion solution (shape of elastic line) of such a link, which is presented in Eq. 20. They left the task of link modeling with an infinite number of modes to their successors. Transversal oscillations defined by Eq. 20 describe the motion of elastic beam to which we assigned an infinite number of DOFs (modes), and which can be described by a mathematical model composed of an infinite number of equations, in the form:

$$
\begin{aligned}
& \widehat{M}_{1, j}+\widehat{\varepsilon}_{1, j}=0 \\
& j=1,2, \ldots, j, \ldots \infty
\end{aligned} .
$$

Dynamics of each mode is described by one equation. The equations in the model 21 are not of equal structure as our contemporaries, authors of numerous works, presently interpret it. We think that the coupling between the modes involved leads to structural diversity among the equations in the model 21. This explanation is of key importance and is necessary for understanding our further discussion. 
Remark II: The symbol “^” denotes generally the quantities that are related to an arbitrary point of the elastic line of the mode, for example: $\widehat{y}_{1,1}, \widehat{x}_{1,1}, \widehat{\varepsilon}_{1,1}$. The same quantities that are not designated by “^” are defined for the mode tip, for example: $y_{1,1}, x_{1,1}, \boldsymbol{\varepsilon}_{1,1}$.

Remark III: Under a mode we understand the presence of coupling between all the modes present in the system. We analyze the system in which the action of coupling forces (inertial, Coriolis', and elasticity forces) exists between the present modes. To differentiate it from "mode shape" or "assumed mode", we could call it a coupled mode or, shorter, in the text to follow, a mode. This yields the difference in the structure of Euler-Bernoulli equations for each mode.

The Bernoulli solution 17-19 describes only partially the nature of motion of real elastic beams. More precisely, it is only one component of motion. Euler-Bernoulli Eqs. 14-21 should be expanded from several aspects in order to be applicable in a broader analysis of elasticity of robot mechanisms. By supplementing these equations with the expressions that come out directly from the motion dynamics of elastic bodies, they become more complex.

As already mentioned, Eqs. 14-20 were defined under the assumption that the elasticity force is opposed only by the inertial force proper. Besides, it is supposed by definition that the motion in Eq. 14 is caused by an external force, suddenly added and then removed. The solution $17-19$ of D. Bernoulli satisfies these assumptions.

The motion of the considered robotic system mode is far more complex than the motion of the body presented in Fig. 8. This means that the equations that describe the robotic system (its elements) must also be more complex than the Eqs. 14-20, formulated by Euler and Bernoulli. This fact is overlooked, and the original equations are widely used in the literature to describe the robotic system motion. This is very inadequate because valuable pieces of information about the complexity of the elastic robotic system motion are thus lost. Hence, it should be especially emphasized the necessity of expanding the source equations for the purpose of modeling robotic systems, and this should be done in the following way:

- $\quad$ based on the known laws of dynamics, Eq. 16 is to be supplemented by all the forces that participate in the formation of the bending moment of the considered mode. It is assumed that the forces of coupling (inertial, Coriolis, and elastic) between the present modes are also involved, which yields structural difference between Eq. 16 in the model 21,

- Equation 17 is to be supplemented by the stationary character of the elastic deformation caused by the forces involved.

\subsection{Equation of the Elastic Line of the First Mode of the First Link}

Let us consider the motion of the first mode of the given link. The link has $n_{1}$ modes in total. The first mode is the bracket (support) of uniformly distributed mass along the mode, loaded by the moment $\widehat{M}_{1,1}$. The load moment $\widehat{M}_{1,1}$ is composed of all the forces acting on the first mode of the link, and these are inertial forces (own and coupled inertia forces of the other modes), centrifugal, gravitational, Coriolis forces (own and coupled), forces due to relative motion of one mode with respect to the other, coupled elasticity forces of the other modes, as well as the force of the environment dynamics, which is via Jacobian matrix transferred to the motion of the first mode. This means that all these forces participate in generating of bending moment that is in forming elastic deformation as well as of the 
elasticity line of the first mode. In that case the model of elastic line of the first mode of the elastic link is of the form of Euler-Bernoulli equation:

$$
\widehat{H}_{1, j} \frac{d^{2} \widehat{y}_{1, j}}{d t^{2}}+\widehat{h}_{1,1}+j_{e 1,1}^{T} F_{u k}+z_{1, j} \cdot \varepsilon_{1}+\widehat{\varepsilon}_{1,1}=0
$$

$j$ - ordinal number of the considered mode, $j=1,2,3 \ldots n_{1}$.

Vectors in Eq. 22 are of the following form:

$\widehat{H}_{1, j}=\left[\widehat{H}_{1,(1,1)} \widehat{H}_{1,(1,2)} \widehat{H}_{1,(1,3)} \ldots . . \widehat{H}_{1,\left(1, n_{1}\right)}\right]$, the vector characterizing the inertia of the first mode. In forming these equations the choice of generalized coordinates can be completely arbitrary. For example here we have chosen the following quantities: $\widehat{y}_{1,1}, \widehat{y}_{1,2}, \widehat{y}_{1,3}, \ldots \widehat{y}_{1, n_{1}}$ to follow the symbols from source Euler-Bernoulli equations. In principle these can be any other quantities as, for example, in [12]. $\frac{\partial^{2} \widehat{y}_{1, j}}{\partial t^{2}}=$ $\left[\begin{array}{lllll}\frac{\partial^{2} \widehat{y}_{1,1}}{\partial t^{2}} & \frac{\partial^{2} \widehat{y}_{1,2}}{\partial t^{2}} & \frac{\partial^{2} \widehat{y}_{1,3}}{\partial t^{2}} & \cdots & \frac{\partial^{2} \widehat{y}_{1, n_{1}}}{\partial t^{2}}\end{array}\right]^{T}$.

$\hat{h}_{1,1}$ - centrifugal, gravitational and Coriolis forces of the first mode. $j_{e 1,1}^{T}=$ $\left[\begin{array}{llll}J_{e 1,(1,1)} & J_{e 1,(1,2)} & J_{e 1,(1,3)} \ldots \ldots J_{e 1,(1,6)}\end{array}\right]$, the first row of the Jacobian matrix serving to map the impact of the dynamic force of contact $F_{u k}$ on the behavior of the first mode.

$$
z_{1, j}=\left[0-\frac{1}{2^{1}}+\frac{1}{2^{2}}-\frac{1}{2^{3}} \ldots . .(-1)^{\left(n_{1}-1\right)} \frac{1}{2^{\left(n_{1}-1\right)}}\right] .
$$

The vector $z_{1, j}$ characterizes the effect of elasticity forces of the other modes on the first mode. The vector $z_{1, j}$ is obtained by modeling different link structures (with one, two, three...modes).

Moment of bending defined for the tip of any mode of the considered link is:

$$
\varepsilon_{1, j}=F_{1, j} \cdot l_{1, j}=C_{s 1, j} \cdot r_{1, j} \cdot l_{1, j}+B_{s 1, j} \cdot \dot{r}_{1, j} \cdot l_{1, j} .
$$

The rigidity and damping characteristic for the tip of any mode is designated as $C_{s 1, j}[\mathrm{~N} / \mathrm{m}]$ and $B_{s 1, j}[N \cdot s / m]$ respectively, maximal deflection is $r_{1, j}$, the mode length is $l_{1, j}$. The vector of bending moments is $\varepsilon_{1}=\left[\begin{array}{lllllll}\varepsilon_{1,1} & \varepsilon_{1,2} & \varepsilon_{1,3} & \varepsilon_{1,4} & \varepsilon_{1,5} & \ldots & \varepsilon_{1, n_{1}}\end{array}\right]^{T}$. Bending moment defined for an arbitrary point of the first mode is: $\hat{\varepsilon}_{1,1}=\beta_{1,1} \cdot \frac{\partial^{2}\left(\hat{y}_{1,1}+\eta_{1,1} \cdot \hat{y}_{1,1}\right)}{\partial \hat{x}_{1,1}^{2}}$.

The force acting on the formation of elastic line of an arbitrary mode of the considered link is $\hat{F}_{1, j}$. Load moment $\hat{M}_{1,1}$ from Eq. 22 is defined as:

$$
\hat{M}_{1,1}=\hat{H}_{1, j} \frac{d^{2} \hat{y}_{1, j}}{d t^{2}}+\hat{h}_{1,1}+j_{e 1,1}^{T} F_{u k}+z_{1, j} \cdot \varepsilon_{1} .
$$

Thus Eq. 22 can be now written in a simpler form:

$$
\hat{M}_{1,1}+\hat{\varepsilon}_{1,1}=0 .
$$

Equation 25 was defined under the assumption that the elasticity moment $\hat{\varepsilon}_{1,1}$ is opposed by the load moment $\hat{M}_{1,1}$, which, among the other forces, encompasses also the coupled elasticity forces of the other modes. In a stationary regime of robotic task realization, the mentioned moments that oppose the elasticity moment $\widehat{\varepsilon}_{1,1}$ continuously change during the 
robotic task realization. Disturbance forces can also act on this system, which may be of an instantaneous or permanent character.

Therefore, elastic deformations of a given body can be generated by:

- disturbance forces, causing oscillatory motion,

- stationary forces, causing stationary motion.

By superimposing the particular solutions of oscillatory character and stationary solution of forced character, any elastic deformation can be presented in the following general form:

$$
\begin{aligned}
& \hat{y}_{1,1}=\hat{X}_{1,1}\left(\hat{x}_{1,1}\right) \cdot\left(\hat{T}_{s t 1,1}(t)+\hat{T}_{t o 1,1}(t)\right)= \\
& =\hat{a}_{1,1}\left(\hat{x}_{1,1}, \hat{T}_{s t 1,1}, \hat{T}_{t o 1,1}, t\right)
\end{aligned}
$$

$\hat{T}_{s t 1,1}(t)$ is the stationary part of elastic deformation caused by stationary forces that may continuously change in time.

$\hat{T}_{t o 1, j}(t)$ is the oscillatory part of elastic deformation as in 19 .

Total motion of the considered mode, defined by the sum of stationary and oscillatory motion, is given by Eq. 26.

Orientation of any point of the first mode is defined by:

$$
\widehat{\psi}_{1,1}=\widehat{d}_{1,1}\left(\widehat{x}_{1,1}, \widehat{T}_{s t 1,1}, \widehat{T}_{t o 1,1}, t\right)
$$

As we have defined the elastic line model of the first mode by Eq. 22 we can also define the model of elastic line of the second, third.... $n_{1}$-th mode of the elastic link.

\subsection{Equation of the Elastic Line of the link}

The elastic line model of the first link that has $n_{1}$ modes is given in a matrix form by the following Euler-Bernoulli equation:

$$
\widehat{H}_{1} \cdot \frac{d^{2} \widehat{y}_{1, j}}{d t^{2}}+\widehat{h}_{1}+j_{e 1}^{T} \cdot F_{u k}+z_{1} \cdot \varepsilon_{1}+\widehat{\varepsilon}_{1}=0
$$

Matrixes and vectors in Eq. 28 are of the following form:

$\widehat{H}_{1} \in R^{n_{1} x n_{1}} \quad$ - the matrix characterizing the inertia of the each mode,

$\widehat{h}_{1} \in R^{n_{1} \times 1} \quad-$ the vector characterizing the effect of centrifugal, gravitational and Coriolis forces of each mode,

$j_{e 1}^{T} \in R^{n_{1} x 6} \quad$ - the Jacobian matrix serving to map the impact of the dynamic force of contact $F_{u k}$ on the behavior of each mode,

$$
z_{1}=\left[\begin{array}{cccccc}
0 & -\frac{1}{2^{1}} & \frac{1}{2^{2}} & -\frac{1}{2^{3}} & \ldots & (-1)^{\left(n_{1}-1\right)} \frac{1}{2^{\left(n_{1}-1\right)}} \\
0 & 0 & -\frac{1}{2^{1}} & \frac{1}{2^{2}} & \ldots & (-1)^{\left(n_{1}-2\right)} \frac{1}{2^{\left(n_{1}-2\right)}} \\
0 & 0 & 0 & -\frac{1}{2^{1}} & \ldots & (-1)^{\left(n_{1}-3\right)} \frac{1}{2^{\left(n_{1}-3\right)}} \\
\ldots & \ldots & \ldots & \ldots & \ldots & \ldots \\
0 & 0 & 0 & 0 & \ldots & 0
\end{array}\right]
$$


Matrix $z_{1}$ characterizes the mutual effect of elasticity forces of the presented modes on the observed mode. Bending moment defined for an arbitrary point of the first link is: $\widehat{\varepsilon}_{1} \in R^{n_{1} x 1}$.

$$
\widehat{\boldsymbol{\varepsilon}}_{1}=\left[\beta_{1,1} \cdot \frac{\partial^{2}\left(\widehat{y}_{1,1}+\eta_{1,1} \cdot \dot{\hat{y}}_{1,1}\right)}{\partial \widehat{x}_{1,1}^{2}} \quad \ldots \beta_{1, n_{1 l}} \cdot \frac{\partial^{2}\left(\widehat{y}_{1, n_{1 l}}+\eta_{1, n_{1 l}} \cdot \dot{\hat{y}}_{1, n_{1 l}}\right)}{\partial \widehat{x}_{1, n_{1 l}}^{2}}\right]^{T} .
$$

Equation 28 represents the equation of motion of elastic line of the first link.

As we have defined the motion of any point on the first mode elastic line by Eqs. 26 and 27 , we can also define the motion of any point on the elastic line of the second, third... $n_{1}$-th mode of the elastic link.

By superimposing the solutions 26 and 27 for all the present modes of the first link and adding to it the dynamics of motor motion that drives it, we obtain total solution of the system in the form:

$$
\begin{aligned}
& \widehat{y}_{1}=R_{1}\left(\bar{\theta}_{1}, t\right)+\sum_{j=1}^{n_{1}} \widehat{X}_{1, j}\left(\widehat{x}_{1, j}\right)\left(\widehat{T}_{s t 1, j}(t)+\widehat{T}_{t o 1, j}(t)\right)= \\
& =\widehat{a}_{1}\left(\widehat{x}_{1, j}, \widehat{T}_{s t 1, j}, \widehat{T}_{t o 1, j}, \bar{\theta}_{1}, t\right)
\end{aligned}
$$

On considering Fig. 9 we can see that the position $\hat{x}_{1}$ should also be defined, which is not only $\sum_{j=1}^{n_{1}} \widehat{x}_{1, j}$ (because the directions of the axes $\widehat{x}_{1,1}, \widehat{x}_{1,2} \ldots \widehat{x}_{1, n_{1}}$ most often do not coincide with the direction of the axis $\hat{x}_{1}$ ), but also includes to a significant extent the geometry and characteristics of the mechanism bending, i.e. the mechanism's dynamics.

$$
\begin{aligned}
& \widehat{x}_{1}=N_{1}\left(\bar{\theta}_{1}, t\right)+\sum_{j=1}^{n_{1}} \widehat{K}_{1, j}\left(\widehat{x}_{1, j}\right)\left(\widehat{T}_{s t 1, j}(t)+\widehat{T}_{t o 1, j}(t)\right)= \\
& =\widehat{b}_{1}\left(\widehat{x}_{1, j}, \widehat{T}_{s t 1, j}, \widehat{T}_{t o 1, j}, \bar{\theta}_{1}, t\right)
\end{aligned}
$$

Any form of elastic line and the pertinent transversal oscillations, as well as the motor motion, can be presented by Eqs. 29 and 30. To this equation one should add also the equation defining the orientation of each point on the elastic line of the link.

$$
\widehat{\psi}_{1}=\widehat{d}_{1}\left(\widehat{x}_{1, j}, \widehat{T}_{s t 1, j}, \widehat{T}_{t o 1, j}, \bar{\theta}_{1}, t\right) \text {. }
$$

In Fig. 9 we sketched the possible forms of elastic line of the $i$-th link having $n_{i}$ modes that appear in the plane $x_{i}-y_{i}$. The plane $x_{i}-y_{i}$ is rotated by the angle $\alpha$, characterizing in the figure the position of the link base with respect to the main coordinate frame $x-y-z$. In the same figure we presented only some of the possible forms of elastic line. The link tip can assume very different positions in the plane $x_{i}-y_{i}$.

\subsection{Equation of the Elastic Line of the Robotic System}

Let us consider a robotic system with $m$ links, whereby the first link contains $n_{1}$ modes, the second link $n_{2}$ modes, ...the $m$-th link contains $n_{m}$ modes. See Fig. 10. Model of the elastic line of this complex elastic robotic system is given in the matrix form by the following Euler-Bernoulli equation:

$$
\widehat{H} \cdot \frac{d^{2} \widehat{y}}{d t^{2}}+\widehat{h}+j_{e}^{T} \cdot F_{u k}+z \cdot \Theta \cdot \varepsilon+\widehat{\varepsilon}=0
$$




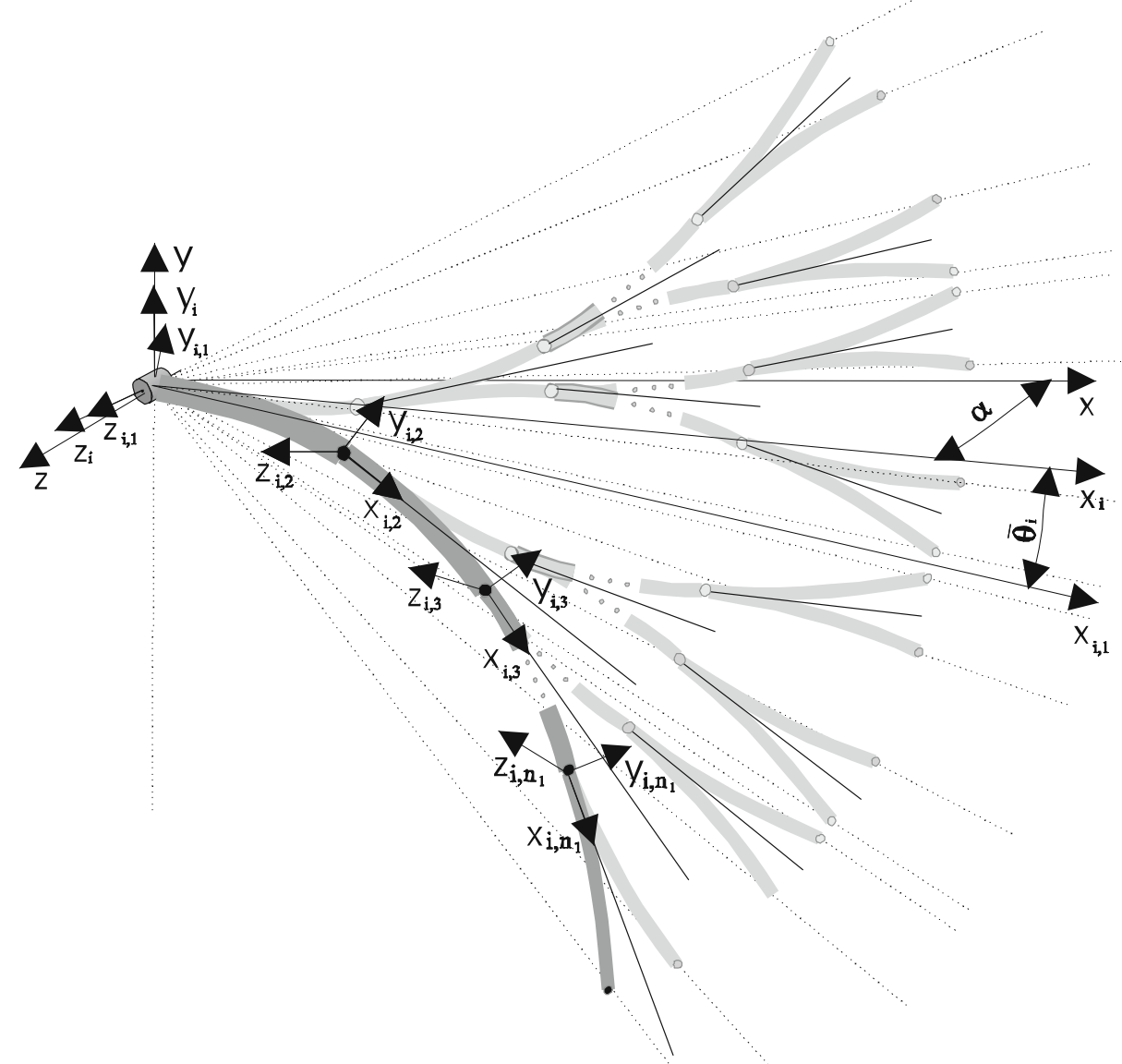

Fig. 9 Possible positions of the tip of elastic line with $n_{1}$ modes

If we define $k=\sum_{i=1}^{m} n_{i}$ then we have that $\widehat{H} \in R^{k x k}$ - matrix characterizing the inertia, $\widehat{h} \in R^{k x 1}$ - vector of the centrifugal, gravitational and Coriolis forces, $j_{e}^{T} \in R^{k x 6}-$ Jacobian matrix mapping the effect of the dynamic contact force $F_{u k}, \Theta \in R^{k x k}$ - matrix characterizing the robot configuration,

$$
z=\left[\begin{array}{cccccc}
0 & -\frac{1}{2^{\top}} & \frac{1}{2^{2}} & -\frac{1}{2^{3}} & \ldots & (-1)^{(k-1)} \frac{1}{2^{(k-1)}} \\
0 & 0 & -\frac{1}{2^{\top}} & \frac{1}{2^{2}} & \ldots & (-1)^{(k-2)} \frac{1}{2^{(k-2)}} \\
0 & 0 & 0 & -\frac{1}{2^{1}} & \ldots & (-1)^{(k-3)} \frac{-1}{2^{(k-3)}} \\
\ldots & \ldots & \ldots & \ldots & \ldots & \ldots \\
0 & 0 & 0 & 0 & \ldots & 0
\end{array}\right]
$$




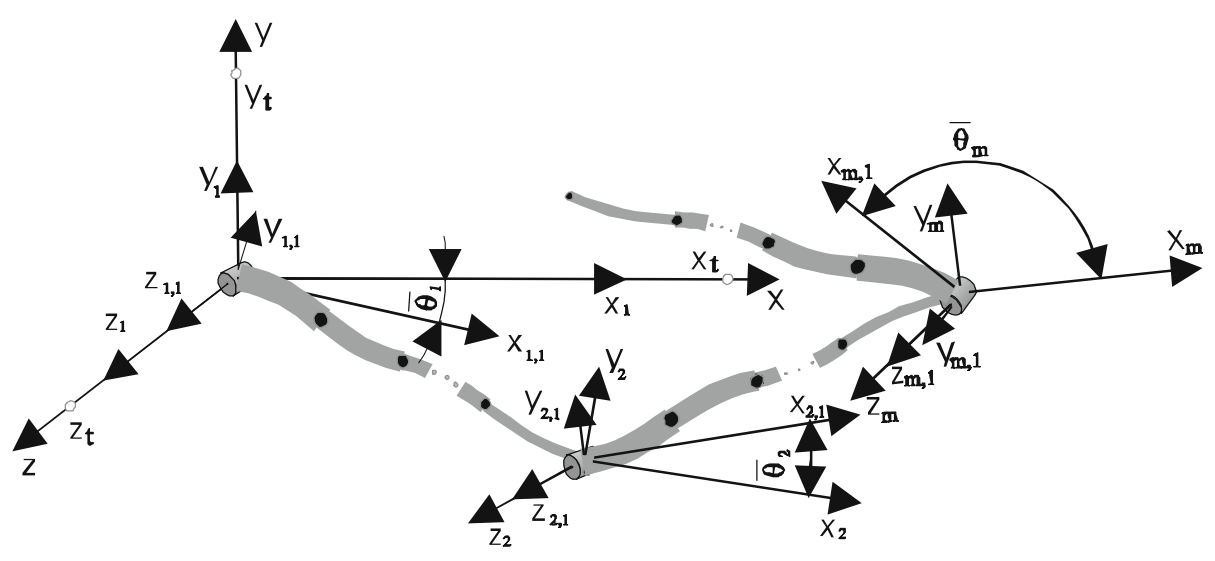

Fig. 10 The shape of elastic line of the complex robotic system with $m$ links

$z \in R^{k x k}$ - matrix characterizing the mutual influence of the forces of elastic modes of all

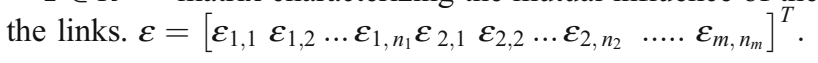

$$
\widehat{\varepsilon}=\left[\beta_{1,1} \cdot \frac{\partial^{2}\left(\widehat{y}_{1,1}+\eta_{1,1} \cdot \dot{\hat{y}}_{1,1}\right)}{\partial \widehat{x}_{1,1}^{2}} \ldots \beta_{n_{m}, n_{m l}} \cdot \frac{\partial^{2}\left(\widehat{y}_{n_{m}, n_{m l}}+\eta_{n_{m}, n_{m l}} \cdot \dot{\hat{y}}_{n_{m}, n_{m l}}\right)}{\partial \widehat{x}_{n_{m}, n_{m l}}^{2}}\right]^{T}
$$

Equation 32 represents the equation of motion of the elastic line of the overall robotic system.

It is known that the robot configuration can substantially influence the mutual position of elastic lines of particular links (see Fig. 10). Solution of the system Eq. 32 and dynamic motor motion, i.e. the form of its elastic line, can be obtained by superimposing the solutions 29-31 for all the links involved in the presence of the dynamics (angle) of rotation of each motor, as well as by taking into account the robotic configuration, i.e. the angle between the axes $z_{i-1}$ and $z_{i}$.

$$
\begin{aligned}
& \widehat{y}=\sum_{i=1}^{m} D_{i}\left(\alpha_{i}\right) \cdot R_{i}\left(\theta_{i}, t\right)+ \\
& +\sum_{i=1}^{m}\left(D_{i}\left(\alpha_{i}\right) \sum_{j=1}^{n_{1}} \widehat{X}_{i, j}\left(\widehat{x}_{i, j}\right)\left(\widehat{T}_{s t i, j}(t)+\widehat{T}_{t o i, j}(t)\right)\right)= \\
& =\widehat{a}\left(\widehat{x}_{i, j}, \widehat{T}_{s t i, j}, \widehat{T}_{t o i, j}, \bar{\theta}, \alpha, t\right) \\
& \widehat{x}=\sum_{i=1}^{m} A_{i}\left(\alpha_{i}\right) \cdot N_{i}\left(\theta_{i}, t\right)+ \\
& +\sum_{i=1}^{m}\left(A_{i}\left(\alpha_{i}\right) \sum_{j=1}^{n_{1}} \widehat{K}_{i, j}\left(\widehat{x}_{i, j}\right)\left(\widehat{T}_{s t i, j}(t)+\widehat{T}_{t o i, j}(t)\right)\right)= \\
& =\widehat{b}\left(\widehat{x}_{i, j}, \widehat{T}_{s t i, j}, \widehat{T}_{t o i, j}, \bar{\theta}, \alpha, t\right)
\end{aligned}
$$




$$
\begin{aligned}
& \widehat{z}=\sum_{i=1}^{m} L_{i}\left(\alpha_{i}\right) \cdot M_{i}\left(\theta_{i}, t\right)+ \\
& +\sum_{i=1}^{m}\left(L_{i}\left(\alpha_{i}\right) \sum_{j=1}^{n_{1}} \widehat{P}_{i, j}\left(\widehat{x}_{i, j}\right)\left(\widehat{T}_{s t i, j}(t)+\widehat{T}_{t o i, j}(t)\right)\right)=. \\
& =\widehat{c}\left(\widehat{x}_{i, j}, \widehat{T}_{s t i, j}, \widehat{T}_{s t i, j}, \bar{\theta}, \alpha, t\right)
\end{aligned}
$$

$D_{i}, A_{i}, L_{i}$ is the function that maps the rotation angle between the axes $z_{i-1}$ and $z_{i} . R_{i}, N_{i}$, $M_{i}$ is the function mapping the rotation angle of the motor shaft $\theta_{i}$ with respect to the $y, x$ and $z$ axis, respectively.

Of course, it is also necessary to define orientation at each point of the elastic line. The orientation of any point of the elastic line of the given robot is defined by:

$$
\begin{aligned}
\widehat{\psi} & =\widehat{d}\left(\widehat{x}_{i, j}, \widehat{T}_{s t i, j}, \widehat{T}_{t o i, j}, \bar{\theta}, \alpha, t\right) . \\
\widehat{\xi} & =\widehat{e}\left(\widehat{x}_{i, j}, \widehat{T}_{s t i, j}, \widehat{T}_{t o i, j}, \bar{\theta}, \alpha, t\right) . \\
\widehat{\varphi} & =\widehat{f}\left(\widehat{x}_{i, j}, \widehat{T}_{s t i, j}, \widehat{T}_{t o i, j}, \bar{\theta}, \alpha, t\right) .
\end{aligned}
$$

Thus we defined the position and orientation of each point of the elastic line in the space of Cartesian coordinates. It should be pointed out that the form of elastic line comes out directly from the dynamics of the system motion.

\section{Relationship Between the Equation of Elastic Line Motion and Equation of Motion at Any Point of the Elastic Line}

Robotics researchers are especially interested in the motion of the first mode tip. At this point act inertial forces (own and the coupled ones of the other modes), centrifugal, gravitational, Coriolis forces (own and coupled), forces due to the relative motion of one mode with respect to the other, coupled elasticity forces of the other modes, as well as the environment force, the effect of the latter on the motion of the considered mode being transferred through the Jacobian matrix. We denote the sum of all these forces by the force $F_{1,1}$ and call it elasticity force.

All the forces forming the force $F_{1,1}$ acting at the distance $I_{1,1}$ from the base of the first mode form the elasticity moment $\varepsilon_{1,1}$ and cause the deflection of the first mode $r_{1,1}$. (see Eq. 23).

The equation of motion of the forces involved at any point of the elastic line of first mode, including the point of the first mode tip, can be defined from the equilibrium equation of elastic line 22. The equation of motion of all forces at the first mode tip for the given boundary conditions can be defined by the following equation:

$$
H_{1, j} \frac{d^{2} y_{1, j}}{d t^{2}}+h_{1,1}+j_{e 1,1}^{T} F_{u k}+z_{1, j} \varepsilon_{1}+\varepsilon_{1,1}=0 \mid \begin{aligned}
& \Sigma F=0 \\
& \text { at the point of } . \\
& \text { first mode tip }
\end{aligned}
$$


Obviously, the term $z_{1, j} \varepsilon_{1}+\varepsilon_{1,1}$ in Eq. 38 could be written in a more compact form, but here we have deliberately written it in a split form, to indicate the presence of elasticity forces of the other modes characterized by $z_{1, j} \varepsilon_{1}$, and which influence the deformation of the considered (first) mode. Equation 39 is interesting because it allows one to calculate the position of the first mode tip. If we know the position of each mode tip we can always calculate the position of the link tip too and eventually the position of the robot tip.

The motion of the mode tip, its position and orientation, are defined by the sum of the stationary and oscillatory motion (cf. Eqs. 26, 27).

$$
\begin{aligned}
& y_{1,1}=a_{1,1}\left(x_{1,1}, T_{s t 1,1}, T_{t o 1,1}, t\right) \mid \begin{array}{l}
\text { tip position of the } \\
\text { first mode in the } \\
\text { direction of the axis } y_{1,1}
\end{array} \\
& \psi_{1,1}=d_{1,1}\left(x_{1,1}, T_{s t 1,1}, T_{t o 1,1}, t\right) \\
& \begin{array}{l}
\text { orientation of the } \\
\text { first mode tip } \\
\text { about the axis } \mathrm{z}_{1,1}
\end{array}
\end{aligned} .
$$

Vector equation of motion of all the forces at the tip of each mode of the first link can be defined from Eq. 28 for the preset boundary conditions:

$$
H_{1} \frac{d^{2} y_{1, j}}{d t^{2}}+h_{1}+j_{e 1}^{T} F_{u k}+z_{1} \varepsilon_{1}+\varepsilon_{1}=0 \quad \begin{aligned}
& \sum F=0 \\
& \text { at the point of } \\
& \text { each mode tip } \\
& \text { of the first link }
\end{aligned}
$$

This equation should be supplemented with the mathematical model of motor.

To describe the behavior of the one-link robotic system having $n_{1}$ modes, the vector Eq. 42 should be supplemented by the mathematical model of the motor. The motor's mathematical model can be defined by writing the equation of motion of all the moments that act on the motor shaft. In the case of a rigid robotic system the motor moment is opposed by the mechanism moment. With elastic robotic systems we have a somewhat different situation: the motor moment is opposed by the bending moment of the first elastic mode that comes after the motor and, partly, by the bending moments of the other elastic modes that are connected in series after the motor. All the modes that come after the motor, due to their position, exert certain influence on the motor dynamics.

The effect of the first mode bending moment is defined by the factor $+\frac{1}{2^{0}}$, of the second by $-\frac{1}{2^{1}}$, of the third by $+\frac{1}{2^{2}}$, of the fourth by $-\frac{1}{2^{3}}$, of the fifth by $+\frac{1}{2^{4}} \cdots$

We add all these elasticity moments to the motor model because they are just to oppose the rotation moment of the motor shaft. The mathematical model of motor is of the following form of equation:

$$
\begin{aligned}
& u_{1}=R_{1} \cdot i_{1}+C_{E 1} \cdot \dot{\bar{\theta}}_{1} \\
& C_{M 1} \cdot i_{1}=I_{1} \cdot \ddot{\bar{\theta}}_{1}+B_{u 1} \cdot \dot{\bar{\theta}}_{1}-S_{1} \cdot\left(z_{m 1, j} \cdot \varepsilon_{1}+\varepsilon_{1,1}\right) \mid \begin{array}{l}
\sum M=0 \\
\text { about the rotation axis } \\
\text { of the first motor }
\end{array}
\end{aligned}
$$


$R_{1}[\Omega]$ is the rotor circuit resistance; $i_{1}[A]$ is the rotor current; $C_{E 1}[\mathrm{~V} /(\mathrm{rad} / \mathrm{s})]$ and $C_{M 1}[\mathrm{Nm} / \mathrm{A}]$ are the proportionality constants of the electromotive force and moment, respectively; $B_{u 1}[\mathrm{Nm} /(\mathrm{rad} / \mathrm{s})]$ is the coefficient of viscous friction; $I_{1}\left[\mathrm{kgm}^{2}\right]$ is the inertia moments of the rotor and reducer; $S_{1}$ is the expression defining the reducer geometry; $\left(z_{m 1, j} \cdot \varepsilon_{1}+\varepsilon_{1.1}\right)$ is the equivalent elasticity moment that opposes the rotation moment of the motor shaft.

$$
z_{m 1, j}=\left[0-\frac{1}{2^{1}}+\frac{1}{2^{2}}-\frac{1}{2^{3}} \ldots . .(-1)^{\left(n_{1}-1\right)} \frac{1}{2^{\left(n_{1}-1\right)}}\right]
$$

The vector $z_{m 1, j}$ characterizes the influence of the elasticity moment of each mode on the motor dynamics.

The overall order of the system $42-43$ is $n_{1}+1$.

The motion of the first link tip is defined by the sum of the stationary and oscillatory motion of the tip of each mode plus the dynamics of motor motion $\bar{\theta}_{1}$ (cf. Eqs. 29-31):

$$
\begin{gathered}
y_{1}=a_{1}\left(x_{1, j}, T_{s t 1, j}, T_{t o 1, j}, \bar{\theta}, t\right) \mid \begin{array}{l}
\text { first link } \\
\text { tip position in the } \\
\text { direction of the axis } y_{1}
\end{array} \\
x_{1}=b_{1}\left(x_{1, j}, T_{s t 1, j}, T_{t o 1, j}, \bar{\theta}, t\right) \\
\psi_{1}=d_{1}\left(x_{1, j}, T_{s t 1, j}, T_{t o 1, j}, \bar{\theta}, t\right) \mid \begin{array}{l}
\text { first link } \\
\text { tip position in the } \\
\text { direction of the axis } x_{1}
\end{array} \\
\begin{array}{l}
\text { tip orientation } \\
\text { about the axis } \mathrm{z}_{1}
\end{array}
\end{gathered}
$$

The equation of motion of all the forces at the point of each mode tip of any link can be defined from Eq. 32 by setting the boundary conditions. Vector equation of all the forces involved for each mode tip of any link is:

$$
H \frac{d^{2} y}{d t^{2}}+h+j_{e}^{T} \cdot F_{u k}+z \cdot \Theta \cdot \varepsilon+\varepsilon=0 \mid \begin{aligned}
& \sum F=0 \\
& \text { at the tip of } \\
& \text { any mode of the } \\
& \text { link considered }
\end{aligned} .
$$

This equation should be supplemented by the vector equation of the mathematical model of motor.

In order to describe the behavior of a robotic system having $m$ links (each of them containing $n_{i}$ modes), we have to add to the vector Eq. 47 the mathematical model of all 
the motors written in a vector form. Let us define it by setting for each motor the equation of motion of all the moments acting about the rotation axis of the given motor. It has the form of the mathematical model of the motor of a rigid robotic system, but the difference being in that the moment of the $i$-th motor is not opposed by the mechanism moment (as with rigid robotic systems). The motor moment is opposed by the bending moment of the first elastic mode that comes after the motor, and also in part, by the bending moments of the other elastic modes that are connected in series after the given motor. All the modes after the motor, due to their position, influence the dynamics of motor motion. Mathematical model of all $m$ motors can be written in a vector form as:

$$
\begin{array}{l|l}
u=R \cdot i+C_{E} \cdot \dot{\bar{\theta}} \\
C_{M} \cdot i=I \cdot \ddot{\bar{\theta}}+B_{u} \cdot \dot{\bar{\theta}}-S \cdot\left(z_{m} \cdot \varepsilon+\varepsilon_{m}\right) & \begin{array}{l}
\sum M=0 \\
\text { about the rotation axis } \\
\text { of the each motors }
\end{array}
\end{array}
$$

In Eq. 48 we have $m$ equations of motors.

$\left(z_{m} \cdot \varepsilon+\varepsilon_{m}\right)$ is the matrix characterizing the effect of elasticity moment of each mode on the motor motion dynamic. $\varepsilon_{m}=\left[\begin{array}{lllll}\varepsilon_{1,1} & \varepsilon_{2,1} & \varepsilon_{3,1} & \ldots . & \varepsilon_{m, 1}\end{array}\right]^{T}$

$z_{m}=\left[\begin{array}{ccrrrr}0 & \ldots & (-1)^{\left(k-n_{m}-1\right)} \frac{1}{2^{\left(k-n_{m}-1\right)}} & (-1)^{\left(k-n_{m}\right)} \frac{1}{2^{\left(k-n_{m}\right)}} & \ldots & (-1)^{(k-1)} \frac{1}{2^{(k-1)}} \\ 0 & \ldots & (-1)^{\left(k-n_{m}-n_{1}-1\right)} \frac{1}{2^{\left(k-n_{m}-n_{1}-1\right)}} & (-1)^{\left(k-n_{m}-n_{1}\right)} \frac{1}{2^{\left(k-n_{m}-n_{1}\right)}} \ldots & (-1)^{\left(k-n_{1}-1\right)} \frac{1}{2^{\left(k-n_{1}-1\right)}} \\ \ldots & \ldots & \ldots & \ldots & \ldots & \ldots \\ 0 & \ldots & \ldots & 0 & \ldots & 0\end{array}\right]$

The overall order of the system $47-48$ is $k+m$.

The robot tip motion is defined by the sum of the stationary and oscillatory motion of each mode tip plus the dynamics of motion of the motor powering each link, as well by the included robot configuration (cf. Eqs. 33-38):

$$
y=a\left(x_{i, j}, T_{s t i, j}, T_{t o i, j}, \bar{\theta}, \alpha, t\right) \mid \begin{aligned}
& \text { tip position of } \\
& \text { the robotic system } \\
& \text { in the direction of the axis } \mathrm{y}
\end{aligned} .
$$

$$
x=b\left(x_{i, j}, T_{s t i, j}, T_{t o i, j}, \bar{\theta}, \alpha, t\right) \mid \begin{aligned}
& \text { tip position of } \\
& \text { the robotic system } \\
& \text { in the direction of the axis } \mathrm{x}
\end{aligned} .
$$

$$
z=c\left(x_{i, j}, T_{s t i, j}, T_{t o i, j}, \bar{\theta}, \alpha, t\right) \mid \begin{aligned}
& \text { tip position of } \\
& \text { the robotic system } \\
& \text { in the direction of the axis } \mathrm{z}
\end{aligned} .
$$




$$
\begin{array}{l|l}
\psi=d\left(x_{i, j}, T_{s t i, j}, T_{t o i, j}, \bar{\theta}, \alpha, t\right) & \begin{array}{l}
\text { tip orientation of } \\
\text { the robotic system } . \\
\text { about the axis z }
\end{array} \\
\xi=e\left(x_{i, j}, T_{s t i, j}, T_{t o i, j}, \bar{\theta}, \alpha, t\right) & \begin{array}{l}
\text { tip orientation of } \\
\text { the robotic system } \\
\text { about the axis y }
\end{array} \\
\varphi=f\left(x_{i, j}, T_{s t i, j}, T_{t o i, j}, \bar{\theta}, \alpha, t\right) & \begin{array}{l}
\text { tip orientation of } \\
\text { the robotic system } \\
\text { about the axis x }
\end{array}
\end{array}
$$

From Eqs. 49-54 we can calculate the position of each mode tip, of each link, and finally, of the robot tip motion.

Generally, we can derive the following conclusion:

To define the form of elastic line of the considered robotic system it is necessary to expand the previously known solutions, namely:

- Supplement it by adding stationary solution to the particular solution of D. Bernoulli, which is of oscillatory character. This means that the given solution depends directly on the overall system dynamics.

- As the link elastic line does not usually conform to the direction of the preset axes but extends in the space, we cannot define it by only one equation. General form of the elastic line is a direct outcome of the dynamics of system motion and cannot be represented by one equation but three equations are needed to define position and three equations to define orientation of each point on the elastic line.

- The equation of elastic line of the robotic system should also encompass the angles of motor shaft rotation $\bar{\theta}$ as in [8], the robot configuration as well, i.e. the angles between the axes $z_{i-1}$ and $z_{i}$.

\section{Reference Trajectory}

There are two aspects in defining the reference trajectory of the motor angle (see [10-12]), viz.:

1) Elastic deformation is considered as a quantity which is not encompassed by the reference trajectory. This is the case when the elasticity characteristics in the system are not known and are not included in the reference trajectory definition. The reference trajectory is defined as for a rigid system.

2) Elastic deformation is a quantity which is at least partly encompassed by the reference trajectory. It is assumed that all elasticity characteristics in the system (both of stiffness and damping) are "known", at least partly and at that level can be included into the process of defining the reference motion. 
Fig. 11 Robotic mechanism

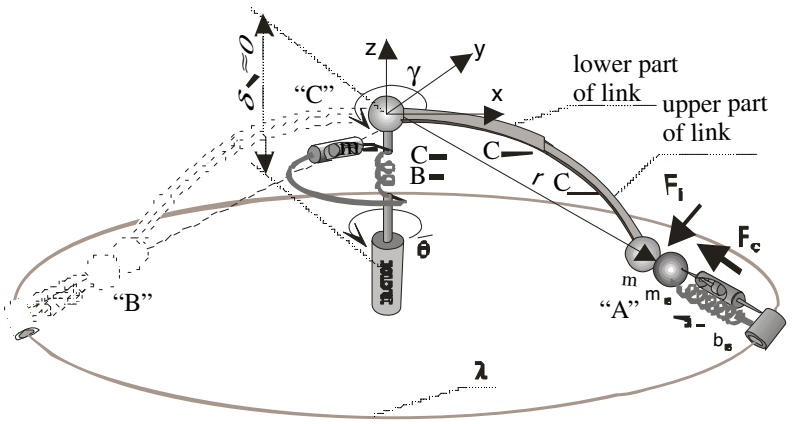

\section{Examples of Simulation}

A robot starts from a point "A" (Fig. 11) and moves toward a point "B" in the predicted time $T=2[s]$. Dynamics of the environment force is included into the dynamics of system's motion [28]. The adopted velocity profile is trapezoidal, with the period of acceleration/ deceleration of $02 \cdot T$.

The same example is analyzed as in paper [12] only with somewhat different parameters flexibility.

Elastic deformation is a quantity which is at least partly encompassed by the reference trajectory as explained in [12] (2.1 under 2).

Example 1: The rigidity characteristic for the tip of first mode is $C_{s 1,1}=6.1569^{*}$ $10^{5}[\mathrm{~N} / \mathrm{m}]$ and $B_{s 1,1}=10[\mathrm{~N} \cdot \mathrm{s} / \mathrm{m}]$ and for the tip of the second mode is $C_{s 1,2}=1.873^{*}$ $10^{4}[\mathrm{~N} / \mathrm{m}]$ and $B_{s 1,2}=600[\mathrm{~N} \cdot \mathrm{s} / \mathrm{m}]$.

All other characteristics of the system and environment are the same as in paper [12].

Fig. 12 Tip coordinates and deviation of position from the reference level (Example 1)

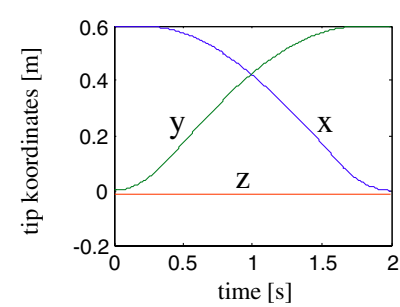

a

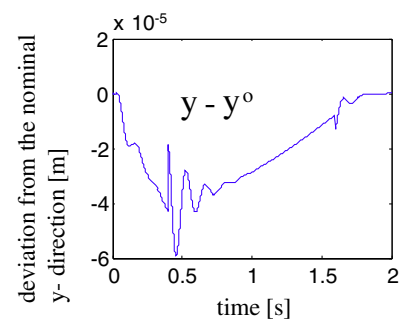

C

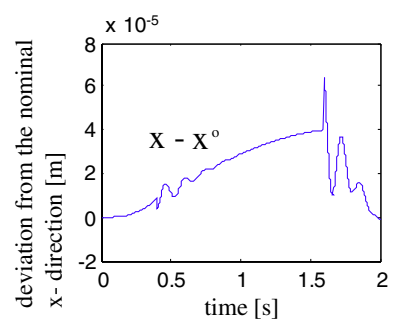

b

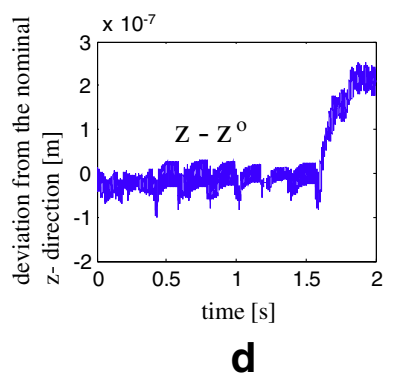


Fig. 13 Dynamic force of the environment (Example 1)

Fig. 14 The elastic deformations (Example 1)
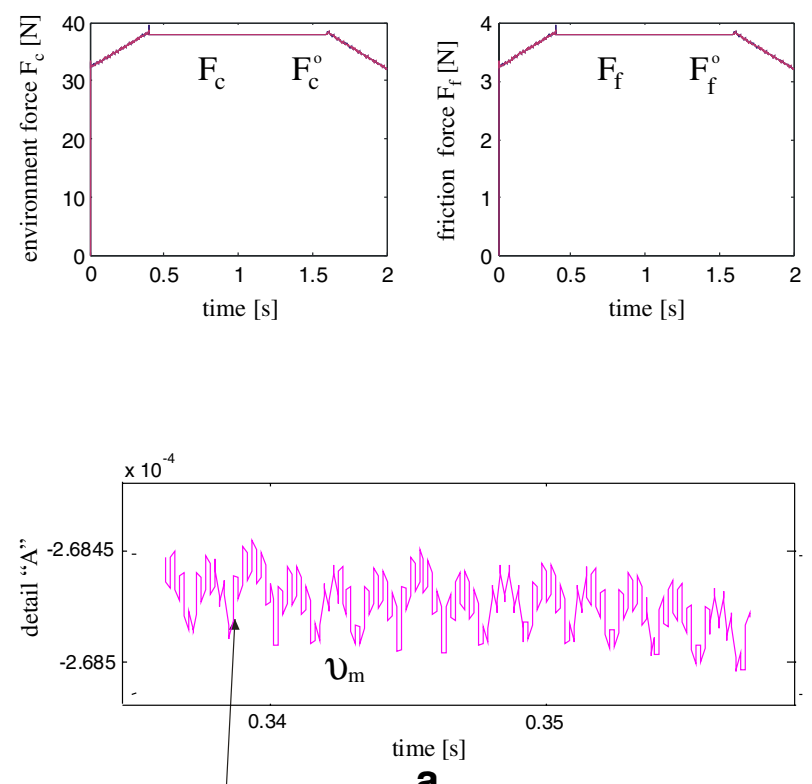

a
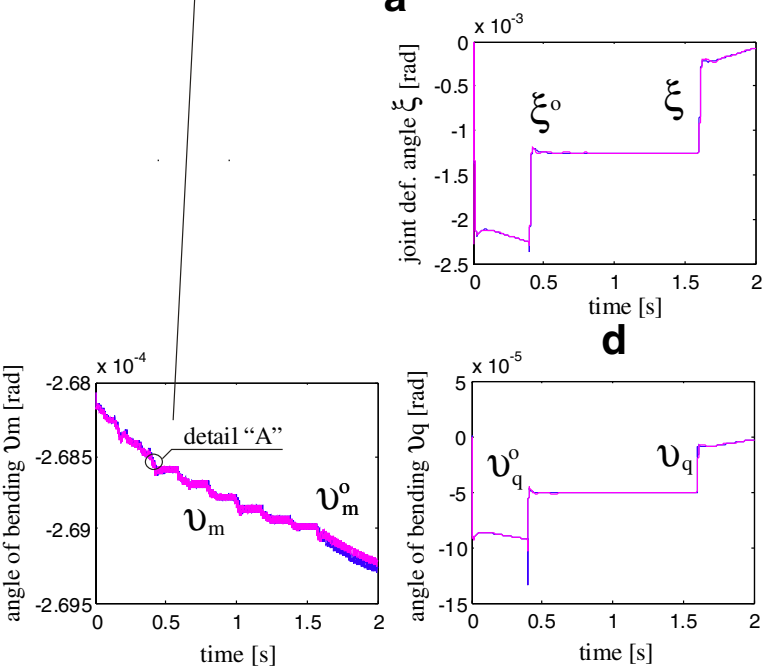

b

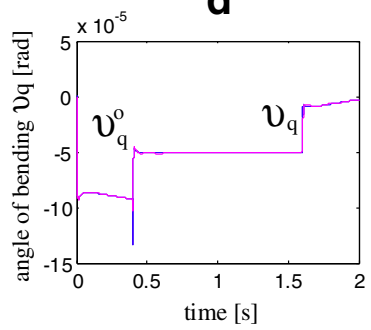

e

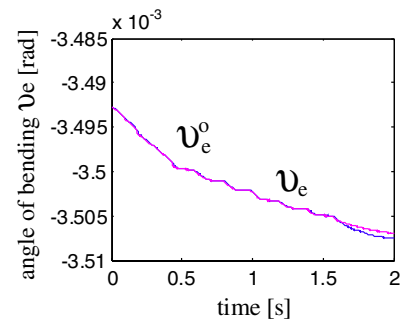

C

in vertical plane

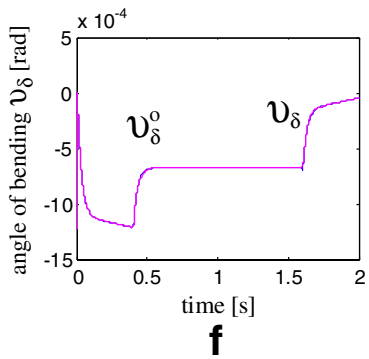

in horizontal plane 
The characteristics of stiffness and damping of the gear in the real and reference regimes are not the same and neither are the stiffness and damping characteristics of the link. $C_{\xi}=0.99 \cdot C_{\xi}^{o}, B_{\xi}=0.99 \cdot B_{\xi}^{o}, C_{s 1,1}=0.99 \cdot C_{s 1,1}^{o}, B_{s 1,1}=0.99 \cdot B_{s 1,1}^{o}, C_{s 1,2}=0.99 \cdot C_{s 1,2}^{o}$, $B_{s 1,2}=0.99 \cdot B_{s 1,2}^{o}$.

As can be seen from Fig. 12 in its motion from point "A" to point "B" the robot tip tracks well the reference trajectory in the space of Cartesian coordinates.

As a position control law for controlling local feedback was applied, the tracking of the reference force was directly dependent on the deviation of position from the reference level (see Fig. 13).

The elastic deformations that are taking place in the vertical plane angle of bending of the lower part of the link (the first mode) $\vartheta_{m}$ and the angle of bending of the upper part of the link (the second mode) $\vartheta_{e}$, as well as elastic deformations taking place in the horizontal plane:, the angle of bending of the lower part of the link (the first mode) $\vartheta_{q}$, the angle of bending of the upper part of the link (the second mode) $\vartheta_{\delta}$ and the deflection angle of gear $\xi$ are given in Fig. 14.

The rigidity of the second mode is about 10 times lower compared with that of the first mode, it is then logical that the bending angle for the second mode is about 10 times larger compared to that of the first mode.

Figure 14a exhibits the wealth of different amplitudes and circular frequencies of the present modes of elastic elements.

Example 2: In contrast to Example 1, the characteristics of stiffness and damping of the gear, first and second mode of the link in the real regime differ significantly from those in the reference regime. $C_{\xi}=0.1 \cdot C_{\xi}^{o}, B_{\xi}=0.1 \cdot B_{\xi}^{o}, C_{s 1,1}=0.1 \cdot C_{s 1,1}^{o}, B_{s 1,1}=0.1 \cdot B_{s 1,1}^{o}$, $C_{s 1,2}=0.1 \cdot C_{s 1,2}^{o}, B_{s 1,2}=0.1 \cdot B_{s 1,2}^{o}$. All other characteristics of the system are the same as in Example 1.

As can be seen from Fig. 15, the real robotic tip motion in the $x, y, z$-directions does not track so well the reference trajectory in the space of Cartesian coordinates as in Example 1. The partial lack of the knowledge of all flexibility characteristics in the robotic system may

Fig. 15 Tip coordinates and deviation of position from the reference level (Example 2)
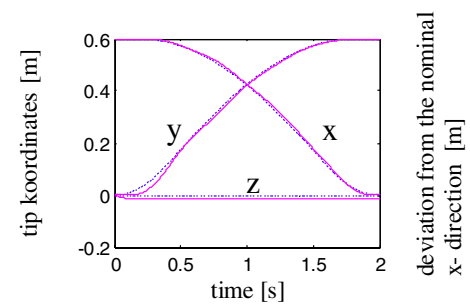

a
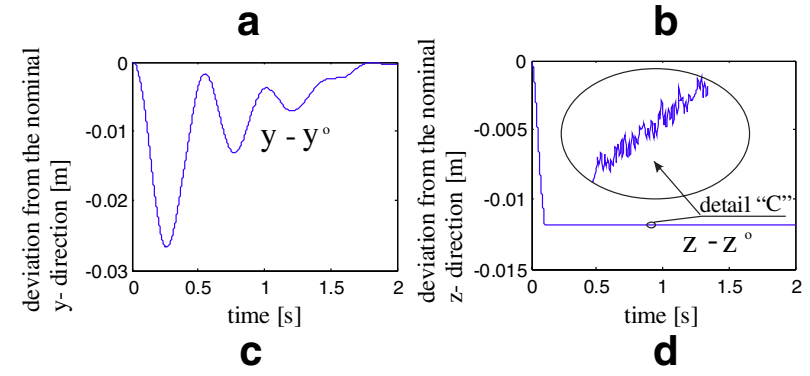
Fig. 16 Dynamic force of the environment (Example 2)

Fig. 17 The elastic deformations (Example 2)
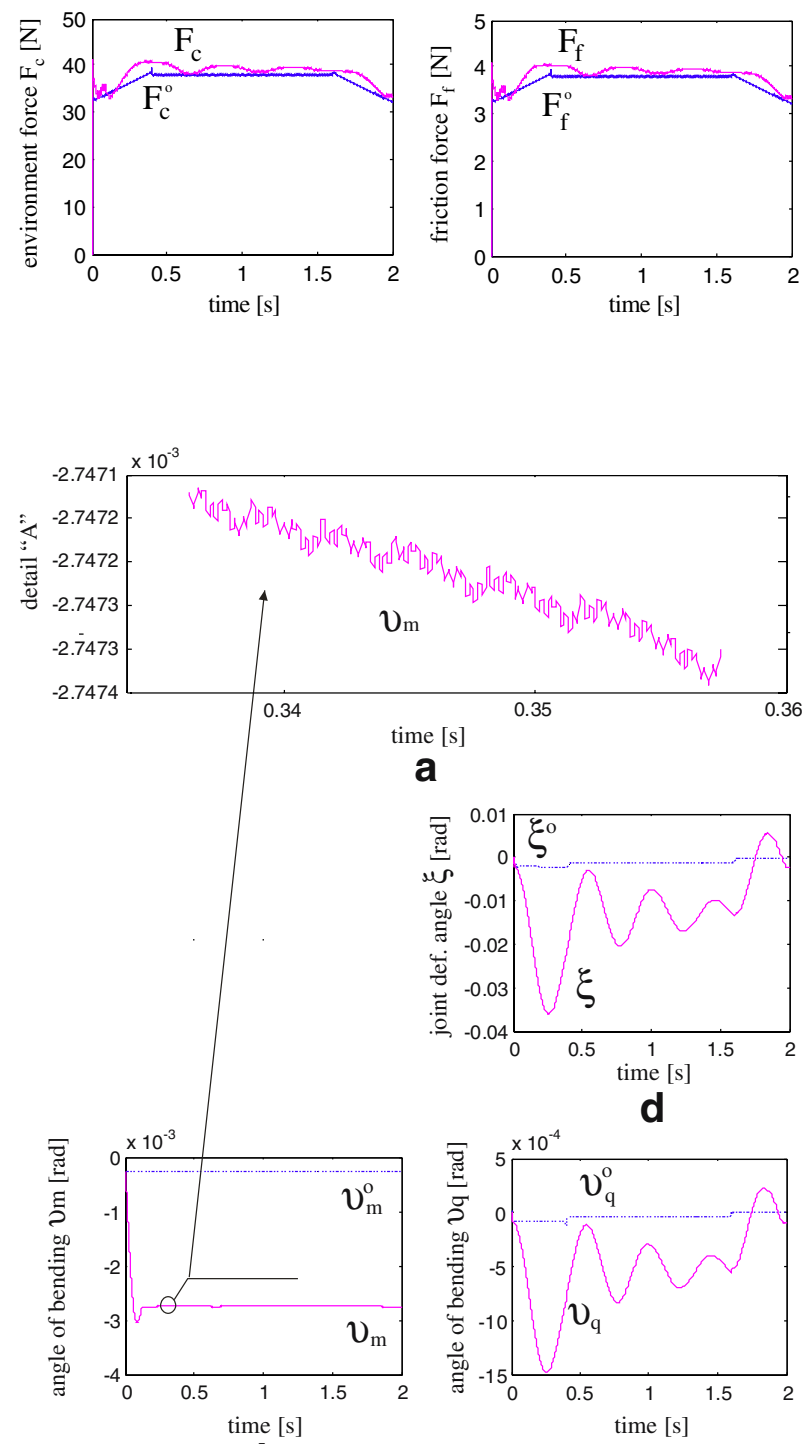

b

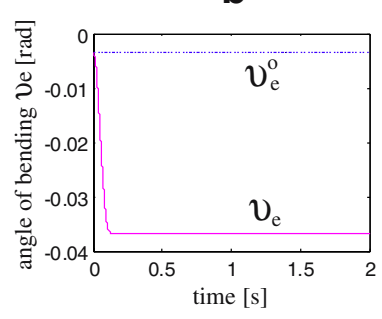

C

in vertical plane

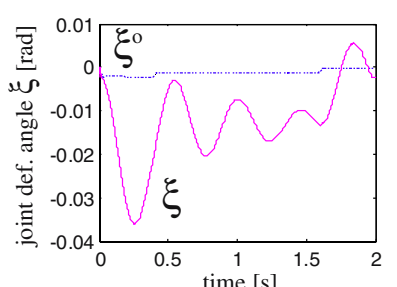

d

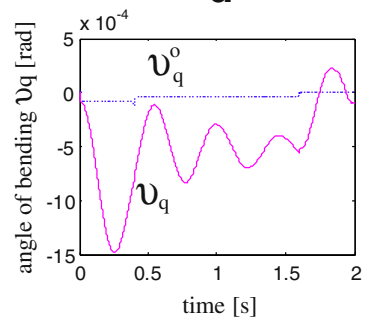

e

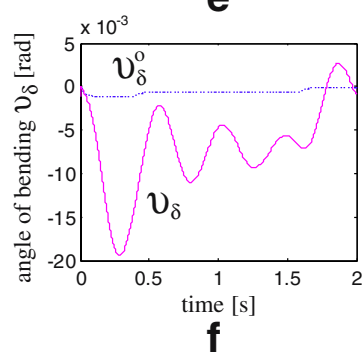

in horizontal plane 
significantly influence the real robotic tip motion, which has now a much larger deviation from the reference trajectory in the space of Cartesian coordinates (cf. Figs. 12 and 15).

In this example, the real force has a more pronounced oscillatory character compared to the reference force (cf. Figs. 13 and 16).

The elastic deformations that take place in the vertical plane: the angle of bending $\vartheta_{m}$ and the angle $\vartheta_{e}$, as well as elastic deformations taking place in the horizontal plane: the gear deflection angle $\xi$, angle of bending $\vartheta_{q}$ and the angle $\vartheta_{\delta}$, are shown in Fig. 17.

A more significant lack of knowledge of flexibility characteristics of all the elastic elements causes larger deviations of this quantity from the reference in the course of robotic task realization. (cf. Figs. 14 and 17).

Let us show the special significance of results from Figs. 14a and 17a. These Figures exhibit the wealth of different amplitudes and circular frequencies of the present modes of elastic elements. We have oscillations within oscillations. This confirms that we have modeled all elastic elements as well as high harmonics (in this case two harmonics of considered link).

\section{Conclusion}

A joint is defined in a new way, in dependence of the motor state (active or locked) and type of elastic or rigid element (gear and/or link) that follows behind the motor. With so defined types of joints that may appear in a robotic construction it is possible to use the known equations to calculate the matrices of transformation and Jacobi matrix. An analysis was made of the choice of reference trajectory, which depends on the level of knowing elasticity characteristics. The estimated elasticity characteristics may be included into the reference trajectory, and thus into the control law.

Based on the EBA, we defined the equation of elastic line of the first mode of any link of a complex robotic system. We have also defined a model of the elastic line of the first link that has $n_{1}$ modes, as well as a mathematical model of motor which moves the same link. On the same principles is defined the model of the elastic line of complex elastic robotic system with $m$ segments, and each segment has $n_{i}$ modes and also the mathematical model of motors which move each link.

We demonstrated that the equation of motion of all the forces involved at any point follows directly from the equation of elastic line. If we define boundary conditions for the mode tip as the most interesting point on the elastic line, we obtain the equation of motion at that point, what is classical form of the mathematical model of the elastic robotic system considered, which essentially LMA is. Thus we demonstrated the connection of the LMA and EBA. LMA is just a special case of EBA. In addition to the comparative analysis of the EBA and LMA, the paper also analyzes a number of other phenomena that make constitutive parts of the motion dynamics of these systems.

a) Euler-Bernoulli equation has been expanded from several aspects:

1) Euler-Bernoulli equation (based on the known laws of dynamics) should be supplemented with all the forces that are participating in the formation of the bending moment of the considered mode, what causes the difference in the structure of these equations for each mode. 
2) Structure of the stiffness matrix must also have the elements outside the diagonal, because of the existence of strong coupling between the elasticity forces involved.

3) Damping is an omnipresent elasticity characteristic of real systems, so that it is naturally included in the Euler-Bernoulli equation.

4) General form of the transversal elastic deformation is defined by superimposing particular solutions of oscillatory character (solution of Daniel Bernoulli) and stationary solution of the forced character (which is a consequence of the forces involved).

5) General form of the elastic line is a direct outcome of the dynamics of system motion and cannot be represented by one scalar equation but three equations are needed to define the position and three equations to define the orientation of each point on the elastic line.

b) Structure of the mathematical models of actuators: With elastic robotic systems, the actuator torque is opposed by the bending moment of the first elastic mode, which comes after the motor, and partly by the bending moments of other modes, which are connected in series after the motor considered. All modes coming after the motor, because of their position, exert influence on the dynamics of motor motion. The mathematical model in our paper is connected to the rest of the mechanism via the equivalent elasticity moment. New structures of the stiffness matrix and mathematical model of actuators appear as a consequence of the coupling between the modes of particular links.

Elastic deformation is a consequence of the overall dynamics of the robotic system, what is essentially different from the method that was used until today, which purports usage of "assumed modes technique". All this has been presented for a relatively simple robotic system that offered the possibility of analyzing the phenomena involved. Through the analysis and modeling of an elastic mechanism we made an attempt to give a contribution to the development of this area.

Open Access This article is distributed under the terms of the Creative Commons Attribution Noncommercial License which permits any noncommercial use, distribution, and reproduction in any medium, provided the original author(s) and source are credited.

\section{References}

1. Bayo, E.: A Finite-element approach to control the End-point motion of a single-link flexible robot. J. Robot. Syst. 4, (1), 63-75 (1987)

2. Book, W.J.: Analysis of Massless Elastic Chains with Servo Controlled Joints. Trans ASME J. Dyn. Syst. Meas. Control 101, 187-192 (1979)

3. Book, W.J.: Recursive Lagrangian dynamics of flexible manipulator arms. Int. J. Rob. Res. 3, (3), 87 101 (1984)

4. Book, W.J., Maizza-Neto, O., Whitney, D.E.: Feedback control of two beam, two joint systems with distributed flexibility. Trans ASME J. Dyn. Syst. Meas. Control 97, (G4), 424-431 (1975)

5. Book, W.J., Majette, M.: Controller design for flexible, distributed parameter mechanical arms via combined state space and frequency domain techniques. Trans ASME J. Dyn. Syst. Meas. Control 105, 245-254 (1983)

6. Cheong, J., Chung, W., Youm, Y.: Bandwidth modulation of rigid subsystem for the class of flexible robots. Proceedings Conference on Robotics \&Automation, San Francisco, pp. 1478-1483, April 2000 
7. Cheong, J., Chung, W.K., Youm, Y.: PID Composite Controller and Its Tuning for Flexible Link Robots. Proceedings of the 2002 IEEE/RSJ, Int. Conference on Intelligent Robots and Systems EPFL. Lausanne, Switzerland (2002) October

8. De Luca, A.: Feedforward/Feedback Laws for the Control of Flexible Robots. IEEE Int. Conf. Robot. Autom. pp 233-240, April 2000.

9. De Luka, A., Siciliano, B.: Closed-form dynamic model of planar multilink lightweight robots. IEEE Trans. Syst. Man Cybern. 21, 826-839 (1991) July/August

10. Filipović, M., Potkonjak, V., Vukobratović, M.: Humanoid robotic system with and without elasticity elements walking on an immobile/mobile platform. J. Intell. Robot. Syst., International Journal 48, 157-186 (2007)

11. Filipović, M., Vukobratović, M.: Modeling of flexible robotic systems, computer as a tool, EUROCON 2005. The International Conference, Belgrade, Serbia and Montenegro 2, 1196-1199 (2005) 21-24 Nov.

12. Filipović, M., Vukobratović, M.: Contribution to modeling of elastic robotic systems. Eng Autom Problems, Int J 5, (1), 22-35 (2006) September 23

13. Ghorbel, F., Spong, W.M.: Adaptive Integral Manifold Control of Flexible Joint Robot Manipulators", IEEE International Conference on Robotics and Automation, Nice, France, May 1992.

14. Hughes, P.C.: Dynamic of a flexible manipulator arm for the space shuttle, AIAA Astrodynamics Conference, Grand Teton National Park. Wyoming, USA (1977)

15. Jang, H., Krishnan, H., Ang, Jr. M.H.: A simple rest-to-rest control command for a flexible link robot. IEEE Int. Conf. on Robotics and Automation, pp 3312-3317, 1997

16. Khadem, S.E., Pirmohammadi, A.A.: Analytical development of dynamic equations of motion for a three-dimensional flexible link manipulator with revolute and prismatic joints. IEEE Trans. Syst. Man Cybern., part B: Cybern. 33(2), (2003), April

17. Kim, J. S., Siuzuki, K., Konno, A., Uchiyama, H.: Force Control of Constrained Flexible Manipulators. Int. Conf. Robot. Autom. 1, 635-640 (1996) April

18. Krishnan, H.: An Approach to Regulation of Contact Force and Position in Flexible-Link Constrained Robots. IEEE, Vancouver (1995)

19. Lord, R.: Theory of Sound, second publish. Paragraph 186, 1894-1896

20. Low, H.K.: A systematic formulation of dynamic equations for robot manipulators with elastic links. J. Robot. Syst. 4, (3), 435-456 (1987)

21. Low, H.K., Vidyasagar, M.: A Lagrangian formulation of the dynamic model for flexible manipulator systems. ASME J. Dyn. Syst. Measurement Control 110, (2), 175-181 (1988) Jun

22. Low, H.K.: Solution schemes for the system equations of flexible robots. J. Robot. Syst. 6, (4), 383-405 (1989) Aug

23. Matsuno, F., Sakawa, Y.: Modeling and Quasi-static hybrid position/force control of constrained planar two-link flexible manipulators. IEEE Trans Robot. Autom. 10(3), (1994), June

24. Matsuno, F., Kanzawa, T.: Robust control of coupled bending and torsional vibrations and contact force of a constrained flexible arm. International Conference on Robotics and Automation, 2444-2449, 1996.

25. Matsuno, F., Wakashiro, K., Ikeda, M.: Force control of a flexible arm. International Conference on Robotics and Automation, 2107-2112, 1994.

26. Meirovitch, L.: Analytical Methods in Vibrations. Macmillan, New York (1967)

27. Moallem, M., Khorasani, K., Patel, V.R.: Tip position tracking of flexible multi-link manipulators: an integral manifold approach. International Conference on Robotics and Automation, 2432-2436, April 1996.

28. Potkonjak, V., Vukobratovic, M.: Dynamics in contact tasks in robotics, Part I general model of robot interacting with dynamic environment. Mech. Mach. Theory 34, 923-942 (1999)

29. Spong, W.M.: Modeling and control of elastic joint robots. ASME J. Dyn. Syst. Measurement Control 109, 310-319 (1987)

30. Spong, W.M.: On the force control problem for flexible joint manipulators. IEEE Trans. Automat. Contr. 34, (1), 107 - 111 (1989) January

31. Surdilovic, D., Vukobratovic, M.: One method for efficient dynamic modeling of flexible manipulators. Mech. Mach. Theory 31, (3), 297-315 (1996)

32. Surdilovic, D., Vukobratovic, M.: Deflection compensation for large flexible manipulators. Mech. Mach. Theory 31, (3), 317-329 (1996)

33. Timoshenko, S., Young, D.H.: Vibration problems in engineering. D. Van Nostrand Company, New York (1955)

34. Vukobratovic, M., Potkonjak, V., Matijević, V.: Control of robots with elastic joints interacting with dynamic environment. J. Intell. Robot. Syst. 23, 87-100 (1998)

35. Yim, W.: Modified nonlinear predictive control of elastic manipulators. International Conference on Robotics and Automation, 2097-2102, April 1996. 